\title{
Understanding nonequilibrium scaling laws governing collapse of a polymer
}

\author{
Suman Majumder ${ }^{\mathrm{a}}$, Henrik Christiansen ${ }^{\mathrm{b}}$, and Wolfhard Janke ${ }^{\mathrm{c}}$ \\ Institut für Theoretische Physik, Universität Leipzig, IPF 231101, 04081 Leipzig, Germany
}

Received 2 October 2019 / Received in final form 6 May 2020

Published online 3 August 2020

(C) The Author(s) 2020. This article is published with open access at Springerlink.com

\begin{abstract}
Recent emerging interest in experiments of single-polymer dynamics urge computational physicists to revive their understandings, particularly in the nonequilibrium context. Here we briefly discuss the currently evolving approaches of investigating the evolution dynamics of homopolymer collapse using computer simulations. Primary focus of these approaches is to understand various dynamical scaling laws related to coarsening and aging during the collapse in space dimension $d=3$, using tools popular in nonequilibrium coarsening dynamics of particle or spin systems. In addition to providing an overview of those results, we also present new preliminary data for $d=2$.
\end{abstract}

\section{Introduction}

Understanding various scaling laws governing a phase transition has been one of the primary research topics over the last fifty years, be it from an equilibrium perspective or at the nonequilibrium front [1-4]. Also for polymers, the equilibrium aspects of phase transitions have been studied extensively [5-8]. Polymers in general represent a large class of macromolecules be they chemically synthesized or naturally occurring. A range of fundamentally important biomolecules, e.g., proteins and DNA, fall under the broad canopy of polymers. Most of these polymeric systems exhibit some form of conformational phase transitions depending on certain external conditions, viz., the collapse transition in homopolymers. Upon changing the solvent condition from good (where monomer-solvent interaction is dominating). to poor (where monomer-monomer interaction is stronger), a homopolymer undergoes a collapse transition from its extended coil state to a compact globule $[9,10]$. This transition belongs to a class of phase transitions that can be understood by investigating various associated scaling laws [5-8]. From a general point of view, the understanding of the collapse transition in homopolymers can be extended to investigate other conformational transitions experienced by different types of macromolecules, e.g., in a protein the collapse of the backbone may occur simultaneously or precede its folding to a native state [11-15].

Due to certain technical difficulties such as preparing a super-dilute solution or finding a long enough

\footnotetext{
${ }^{a}$ e-mail: suman.majumder@itp.uni-leipzig.de

b e-mail: henrik.christiansen@itp.uni-leipzig.de

${ }^{c}$ e-mail: wolfhard.janke@itp.uni-leipzig.de
}

polymer with negligible polydispersity, the experimental realization of the collapse transition was rare in the past $[10,16]$. Since the introduction of technical equipment like small angle X-ray scattering, single molecule fluorescence, dynamic light scattering, dielectric spectroscopy, etc., monitoring the behaviour of a single macromolecule has become feasible [17-19]. On the other hand, theoretically the scaling laws related to the static and the equilibrium dynamic aspects of the transition are well understood since a long time [5-8].

In contrast to the equilibrium literature, however, in the nonequilibrium aspects, i.e., for the kinetics of the collapse transition, there is no unanimous theoretical understanding even though quite a few analytical and computational studies have been conducted [20-35]. The aforesaid experimental developments to track single polymers and the lack of understanding of the nonequilibrium dynamics of polymers motivated us to perform a series of works on the kinetics of polymer collapse [36-41]. There our novel approach of understanding the collapse by using its analogy with usual coarsening phenomena of particle and spin systems provided intriguing new insights, as will be discussed subsequently.

Most of the studies on collapse kinetics in the past dealt with the understanding of the relaxation time, i.e., the time a system requires to attain its new equilibrium state once its current state is perturbed by a sudden change of the environmental conditions, e.g., the temperature. In the context of polymer collapse, the relaxation time is referred to as the collapse time $\tau_{c}$, which measures the time a polymer that is initially in an extended state needs to reach its collapsed globular phase. Obviously, $\tau_{c}$ depends on the degree of polymerization or chain length $N$ of the polymer (the number of repeating units 
or monomers in the chain), which can be described by the scaling relation

$$
\tau_{c} \sim N^{z},
$$

where $z$ is the corresponding dynamical exponent. The above relation is reminiscent of the scaling one observes for dynamic critical phenomena [42]. The other important aspect of the kinetics is the growth of clusters of monomers that are formed during the collapse [21,31]. The cluster growth has recently been understood by us using the phenomenological similarities of collapse with coarsening phenomena in general $[36,39,40]$. Moreover, along the same line one can also find evidence of aging and related scaling laws [37-40] that was mostly ignored in the past.

In this Colloquium, we intend to give a brief review of the results available on collapse kinetics based on the above mentioned three topics: relaxation, coarsening, and aging. It is organized in the following way. We will begin with an overview of the phenomenological theories of collapse dynamics followed by an overview of the previous simulation results in Section 2. Afterwards, in Section 3, we will discuss our recent developments concerning the understanding of relaxation time, cluster growth and aging for the kinetics of the collapse transition in a homopolymer. Then we will present in Section 4 some preliminary results on the special case of polymer collapse kinetics in space dimension $d=2$. In Section 5, finally, we wrap up with a discussion and an outlook to future research in this direction.

\section{Overview of previous studies on collapse dynamics}

The first work on the collapse dynamics dates back to 1985 when de Gennes proposed the phenomenological sausage model [20]. It states that the collapse of a homopolymer proceeds via the formation of a sausage-like intermediate structure which eventually minimizes its surface energy through hydrodynamic dissipation and finally forms a compact globule having a spherical shape. Guided by this picture, in the next decade there was a series of numerical works by Dawson and co-workers considering both lattice and off-lattice models [21-26]. However, the sequence of events obtained in their simulations differs substantially from the sausage model. Later in 2000, Halperin and Goldbart (HG) came up with their pearl-necklace picture of the collapse [29], consistent not only with the observations of Dawson and co-workers but also with all the later simulation results. According to HG the collapse of a polymer upon quenching from an extended coil state into the globular phase occurs in three different stages: (i) initial stage of formation of many small nascent clusters of monomers out of the density fluctuations along the chain, (ii) growth and coarsening of the clusters by withdrawing monomers from the bridges connecting the clusters until they coalesce with each other to form bigger clusters and eventually ending up with a single cluster, and (iii) the final stage of rearrangements of the monomers within the single cluster to form a compact globule. Even before the pearl-necklace picture of collapse by HG, Klushin [28] independently proposed a phenomenology for the same picture based on similar coarsening of local clusters. It differs from the HG one as it does not consider the initial stage of formation of the local ordering or small nascent clusters. However, almost all the simulation results so far have shown evidence for the initial stage of nascent cluster formation.

In addition to the above description, HG also provided time scales for each of these stages which scale with the number of monomers as $N^{0}, N^{1 / 5}$ and $N^{6 / 5}$, respectively. Quite obviously this scaling of the collapse time is dependent on the underlying dynamics of the system, i.e., on the consideration of hydrodynamic effects. Klushin derived that the collapse time $\tau_{c}$ scales as $\tau_{c} \sim N^{1.6}$ in absence of hydrodynamics whereas the collapse is much faster in presence of hydrodynamics with the scaling $\tau_{c} \sim N^{0.93}$ [28]. Similar conclusions were drawn in other theoretical and simulation studies as well. In the following Section 2.1 we discuss some of these numerical results on the scaling of the collapse time.

\subsection{Earlier results on scaling of collapse time}

As mentioned the dynamical exponent $z$ in equation (1) depends on the intrinsic dynamics of the system. It is thus important to notice the method and even the type of model one uses for the computer simulations. The available results can be divided into three categories: (i) Monte Carlo (MC) and Langevin simulations with implicit solvent effect, (ii) molecular dynamics (MD) simulations with implicit solvent effect, and (iii) MD simulations with explicit solvent effect. Results from MC and Langevin simulations do not incorporate hydrodynamics and hence only mimic diffusive dynamics. On the other hand, MD simulations with implicit solvent, depending on the nature of the thermostat used for controlling the temperature, can be with or without hydrodynamic effects. At this point we caution the reader that there is a subtle difference between solvent effects and hydrodynamic effects. Thus doing MD simulations with explicit solvent does not necessarily mean that the hydrodynamic modes are actively taken into account. Rather this depends on how one treats the momenta of the solvent particles in the simulation, e.g., it depends on the choice of thermostat used [43]. This gets not only reflected in the nonequilibrium relaxation times like the collapse time $\tau_{c}$ but also in the equilibrium autocorrelation time $\tau$. The few existing studies on polymer collapse using MD simulations that account for solvent effects by considering explicit solvent beads, thus, can also be classified on the basis of consideration of hydrodynamic effects. Since there is no available appropriate theory for the nonequilibrium relaxation time, the trend is to compare the scaling of the collapse time with the available theories of equilibrium polymer dynamics. In absence of hydrodynamic effects the dynamics is compared with Rouse scaling that states that in equilibrium the diffusion coefficient $D$ scales with the chain length $N$ as $D \sim N^{-1}$, which implies that the relaxation time scales as $\tau \sim N^{2}$ [44]. On the other hand, in presence of hydrodynamics when the polymer moves as a whole due to the flow 
Table 1. Summary of simulation results for the scaling of the collapse time $\tau_{c}$ with the length of the polymer $N$ as described in equation (1).

\begin{tabular}{llllll}
\hline Authors & Model & Method & Explicit Solvent & Hydrodynamics & $z$ \\
\hline Byrne et al. (1995) [21] & Off-lattice & Langevin & No & No & $3 / 2$ \\
Kuznetsov et al. (1995) [23] & Lattice & MC simulations & No & No & 2 \\
Kuznetsov et al. (1996) [24] & GSC equations & Numerically & No & No & 2 \\
Kuznetsov et al. (1996) [24] & GSC equations & Numerically & No & Yes & $3 / 2$ \\
Kikuchi et al. (2005) [33] & Off-lattice & MD simulations & Yes & No & $1.89(9)$ \\
Kikuchi et al. (2005) [33] & Off-lattice & MD simulations & Yes & Yes & $1.40(8)$ \\
Pham et al. (2008) [34] & Off-lattice & BD simulations & No & No & $1.35(1)$ \\
Pham et al. (2008) [34] & Off-lattice & BD simulations & No & Yes & $1.01(1)$ \\
Guo et al. (2011) [35] & Off-lattice & DPD simulations & Yes & Yes & $0.98(9)$ \\
Majumder et al. (2017) [39] & Off-lattice & MC simulations & No & No & $1.79(6)$ \\
Christiansen et al. (2017) [40] & Lattice & MC simulations & No & No & $1.61(5)$ \\
\hline
\end{tabular}

MC: Monte Carlo, MD: molecular dynamics, BD: Brownian dynamics, DPD: dissipative particle dynamics.

field, the corresponding scaling laws are $D \sim N^{-0.588}$ and $\tau \sim N^{1.76}$, known as the Zimm scaling [45]. Both Rouse and Zimm scalings have been verified in a number of computational studies as well as in experiments. However, we stress that the nonequilibrium relaxation time, e.g., the collapse time $\tau_{c}$ does not necessary follow the same scaling as the equilibrium autocorrelation time $\tau[46,47]$.

In Table 1 we have summarized some of the relevant results on the scaling of the collapse time that one can find in the literature. In the early days the simulations were done mostly by using methods that do not incorporate hydrodynamics, e.g., numerical solution of the Gaussian-self consistent (GSC) equations, MC simulations and Langevin simulations. They considered models which could be either on-lattice (interacting self-avoiding walks) or off-lattice (with Lennard-Jones kind of interaction). The GSC approach and MC simulations (in a lattice model) provided $z$ that matches with the Rouse scaling in equilibrium $[23,24]$. Langevin simulations of an off-lattice model yielded $z \approx 3 / 2[21]$ which was the value later obtained in a theory by Abrams et al. [31]. Kikuchi et al. [30] went a step further by doing MD simulations of an off-lattice model with explicit solvent which also allows one to tune the hydrodynamic interactions. In absence of hydrodynamics they obtained values of $z \approx 1.9$ close to the Rouse value of 2 [33]. On the other hand, in presence of hydrodynamics the dynamics is much faster with $z \approx 1.4$ [33]. This is more or less in agreement with GSC results obtained considering hydrodynamic interaction [24]. Later more simulations on polymer collapse with explicit solvent were performed. In this regard, relatively recent Brownian dynamics (BD) simulations with explicit solvent (hydrodynamic interaction preserved) by Pham et al. provided even faster dynamics with $z \approx 1$ [34]. There exist even newer results from dissipative-particle dynamics (DPD) simulation that also reports $z \approx 1$ [35]. Note that these results do not well compare with the Zimm scaling applicable to equilibrium dynamics in presence of hydrodynamics. The bottom line from this literature survey is that no consensus has been achieved for the value of $z$. In our recent results on collapse dynamics from MC simulations a consistent value of $z$ was obtained between an off-lattice model and a lattice model with $z \approx 1.7[39,40]$.

\subsection{Earlier results on cluster growth}

As discussed above most of the previous studies on kinetics of the collapse transition focused on understanding the scaling of the collapse time. However, going by the phenomenological picture described by HG, as also observed in most of the available simulation results, the second stage of the collapse, i.e., the coalescence of the "pearl-like" clusters to form bigger clusters and thereby eventually a single globule bears resemblance to usual coarsening of particle or spin systems. The nonequilibrium phenomenon of coarsening in particle or spin systems is well understood $[4,48]$ with current focus shifting towards more challenging scenarios like fluid mixtures $[49,50]$. Fundamentally, too, it is still developing as for example in computationally expensive long-range systems [51-53].

In usual coarsening phenomena, e.g., in ordering of ferromagnets after quenching from the high-temperature disordered phase to a temperature below the critical point, the nonequilibrium pathway is described by a growing length scale, i.e., average linear size of the domains $\ell(t)$ as $[4,48]$

$$
\ell(t) \sim t^{\alpha} .
$$

The value of the growth exponent $\alpha$ depends on the concerned system as well as the conservation of the order parameter during the entire process. For example, in solid binary mixtures where the dynamics is conserved, $\alpha=1 / 3$ which is the Lifshitz-Slyozov (LS) growth exponent [54], whereas for a ferromagnetic ordering where the order parameter is not conserved, $\alpha=1 / 2$ which is referred to as the Lifshitz-Cahn-Allen (LCA) growth [55]. On the other hand, in fluids where in simulations one must incorporate hydrodynamics, three different regimes are observed; the early-time diffusive growth where $\alpha=1 / 3$ as in solids; the intermediate viscous hydrodynamic growth with $\alpha=1$ [56]; and at a very late stage the inertial growth with $\alpha=2 / 3[57]$.

In the context of polymer collapse, the concerned growing length scale could be the linear size (or radius) of the clusters. However, in all the previous works it was chosen to be the average mass $C_{s}(t)$, or average number of 
monomers present in a cluster. In spatial dimension $d$, it is related to the linear size of the cluster as $C_{s}(t) \sim \ell(t)^{d}$. Thus in analogy with the power-law scaling (2) of the length scale during coarsening, the corresponding scaling of the cluster growth can then be written as

$$
C_{s}(t) \sim t^{\alpha_{c}}
$$

where $\alpha_{c}=d \alpha$ is the corresponding growth exponent. Like the dynamical exponent $z$, the growth exponent $\alpha_{c}$ is also dependent on the intrinsic dynamics of the system. Previous studies based on MC simulations of a lattice polymer model reported $\alpha_{c}=1 / 2$ [23] and Langevin simulations of an off-lattice model reported $\alpha_{c}=2 / 3$ [21], both being much smaller than $\alpha_{c}=1$ as observed for coarsening with only diffusive dynamics. BD simulations with explicit solvent also provided $\alpha_{c} \approx 2 / 3$ in absence of hydrodynamics. Like in coarsening of fluids, the dynamics of cluster growth during collapse, too, gets faster when hydrodynamic effects are present. For instance, BD and DPD simulations with incorporation of hydrodynamic effects yield $\alpha_{c} \approx 1$ [34,35]. Surprisingly, our recent result on an off-lattice model via $\mathrm{MC}$ simulations also showed $\alpha_{c} \approx 1$ [39]. This will be discussed in Section 3.4.

\subsection{Earlier results on aging during collapse}

Apart from the scaling of the growth of the average domain size during a coarsening process there is another important aspect, namely, aging [58,59]. The fact that a younger system relaxes faster than an older one forms the foundation of aging in general. This is also an essential concept from the point of view of glassy dynamics [60,61]. Generally, aging is probed by the autocorrelation function of a local observable $O_{i}$ given as

$$
C\left(t, t_{w}\right)=\left\langle O_{i}(t) O_{i}\left(t_{w}\right)\right\rangle-\left\langle O_{i}(t)\right\rangle\left\langle O_{i}\left(t_{w}\right)\right\rangle,
$$

with $t$ and $t_{w}<t$ being the observation and the waiting time, respectively. The $\langle\ldots\rangle$ denotes averaging over several randomly chosen realizations of the initial configuration and independent time evolutions. The observable $O_{i}$ is generally chosen in such a way that it clearly reflects the changes happening during the concerned nonequilibrium process, e.g., the time- and space-dependent order parameter during ferromagnetic ordering.

There are three necessary conditions for aging: (i) absence of time-translation invariance in $C\left(t, t_{w}\right)$, (ii) slow relaxation, i.e., the relaxation times obtained from the decay of $C\left(t, t_{w}\right)$ should increase as function of $t_{w}$, and (iii) the observation of dynamical scaling of the form

$$
C\left(t, t_{w}\right) \sim x_{c}^{-\lambda} ; x_{c}=\ell / \ell_{w}
$$

where $\ell$ and $\ell_{w}$ are the characteristic length scales at time $t$ and $t_{w}$, respectively, and $\lambda$ is the corresponding autocorrelation exponent. For the choice $x_{c}=t / t_{w}$, the corresponding autocorrelation exponent is $\alpha \lambda$ where the growth exponent $\alpha$ relates $\ell$ and $t$ [see Eq. (2)]. Fisher and Huse $(\mathrm{FH})$ in their study of ordering spin glasses proposed a bound on $\lambda$ which only depends on the dimension $d$ as [62]

$$
\frac{d}{2} \leq \lambda \leq d
$$

Later this bound was found to be obeyed in the ferromagnetic ordering as well [63-65]. An even stricter and more general bound was later proposed by Yeung et al. [66] that also includes the case of conserved order-parameter dynamics.

In the context of polymer collapse, although analogous to coarsening phenomena in general, this particular aspect of aging has received very rare attention $[67,68]$. There, like in other soft-matter systems [69-71] the results indicated presence of subaging, i.e., evidence for scaling similar to equation (5) but as a function of $x_{c}=t / t_{w}^{\mu}$ with $\mu<1$. Afterwards, there were no attempts to quantify this scaling with respect to the ratio of the growing length scale. In our approach, both with off-lattice and lattice models we showed that simple aging scaling as in equation (5) with respect to the ratio of the cluster sizes can be observed [37-40]. Thus to quantify the aging scaling, by choosing $x_{c}=C_{s}(t) / C_{s}\left(t_{w}\right)$ one has to transform equation (5) to

$$
C\left(t, t_{w}\right) \sim\left[\frac{C_{s}(t)}{C_{s}\left(t_{w}\right)}\right]^{-\lambda_{c}}
$$

where $\lambda_{c}$ is the associated autocorrelation exponent which is related to the traditional exponent $\lambda$ via the relation $\lambda_{c}=\lambda / d$.

\section{Recent Monte Carlo results in $d=3$}

In this section we will review the very recent developments by us concerning the kinetics of homopolymer collapse from all above mentioned three perspectives. We will compare the results from an off-lattice model (OLM) and a lattice model (LM), focusing in this section on $d=3$ dimensions. New results for the special case of $d=2$ will be presented in the next section to check the validity of the observations in general. Before moving on to a discussion of our findings next we first briefly describe the different models and methodologies used in our studies.

\subsection{Models and methods}

For OLM, we consider a flexible bead-spring model where the connectivity between two successive monomers or beads is maintained via the standard finitely extensible non-linear elastic (FENE) potential

$$
E_{\mathrm{FENE}}\left(r_{i i+1}\right)=-\frac{K}{2} R^{2} \ln \left[1-\left(\frac{r_{i i+1}-r_{0}}{R}\right)^{2}\right]
$$

We chose the force constant of the spring $K=40$, the mean bond length $r_{0}=0.7$ and the maximum allowed deviation from the mean position $R=0.3$ [72]. Monomers 
were considered to be spherical beads with diameter $\sigma=r_{0} / 2^{1 / 6}$. The nonbonded interaction between the monomers is given by

$$
E_{\mathrm{nb}}\left(r_{i j}\right)=E_{\mathrm{LJ}}\left(\min \left[r_{i j}, r_{c}\right]\right)-E_{\mathrm{LJ}}\left(r_{c}\right),
$$

where

$$
E_{\mathrm{LJ}}(r)=4 \epsilon\left[\left(\frac{\sigma}{r}\right)^{12}-\left(\frac{\sigma}{r}\right)^{6}\right]
$$

is the standard Lennard-Jones (LJ) potential. Here $\epsilon(=1)$ is the interaction strength and $r_{c}=2.5 \sigma$ the cut-off radius.

For LM, we consider a variant of the interactive selfavoiding walk on a simple-cubic lattice, where each lattice site can be occupied by a single monomer. The Hamiltonian is given by

$$
H=-\frac{1}{2} \sum_{i \neq j, j \pm 1} w\left(r_{i j}\right), \text { where } w\left(r_{i j}\right)=\left\{\begin{array}{ll}
J & r_{i j}=1 \\
0 & \text { else }
\end{array} .\right.
$$

Here $r_{i j}$ is the distance between two nonbonded monomers $i$ and $j, w\left(r_{i j}\right)$ is an interaction parameter that considers only nearest neighbours, and $J(=1)$ is the interaction strength. We allowed a fluctuation in the bond length by considering diagonal bonds, i.e., the possible bond lengths are $1, \sqrt{2}$ and $\sqrt{3}$. The model has been independently studied for equilibrium properties [73,74]. It has certain similarities with the bond-fluctuation model [75]. For a comparison between them, please see reference [76].

The dynamics in the models can be introduced via Markov chain MC simulations [46,77], however, with the restriction of allowing only local moves. For OLM the local moves correspond to shifts of a randomly selected monomer to a new position randomly chosen within $[-\sigma / 10: \sigma / 10]$ of its current position. For LM, too, the move set consists of just shifting a randomly chosen monomer to another lattice site such that the bond connectivity constraint is maintained. These moves are then accepted or rejected following the Metropolis algorithm with Boltzmann criterion [46,77]. The time scale of the simulations is one MC sweep (MCS) which consists of $N$ (where $N$ is the number of monomers in the chain) such attempted moves.

The collapse transition temperature is $T_{\theta}(N \rightarrow \infty)$ $\approx 2.65 \epsilon / k_{B}$ and $\approx 4.0 \mathrm{~J} / k_{B}$ for OLM and $\mathrm{LM}$, respectively $[39,40]$. In all the subsequent discussion, the unit of temperature will always be $\epsilon / k_{B}$ or $J / k_{B}$ with the Boltzmann constant $k_{B}$ being set to unity. Following the standard protocol of nonequilibrium studies we first prepared initial conformations of the polymers at high temperature $T_{h} \approx 1.5 T_{\theta}$ that mimics an extended coil phase. Then this high-temperature conformation was quenched to a temperature $T_{q}<T_{\theta}$. Since LM is computationally less expensive than OLM, the chain length of polymer used for LM is longer than what is used for OLM. Note that except for the evolution snapshots, for both models, all the results presented were obtained after averaging over more than 300 independent runs. For each such run, the starting conformation is an extended coil which were obtained independently of each other by generating selfavoiding walks using different random seeds and then equilibrating them at the high temperature $T_{h}$.

\subsection{Phenomenological picture of the collapse}

As mentioned before even though the sausage picture of de Gennes [20] is the pioneer in describing the phenomenology of the collapse dynamics, all simulation studies provided evidence in support of the pearl-necklace picture of HG [29]. In our simulations, too, both with OLM and LM, we observed intermediates that support the pearl-necklace phenomenology. Typical snapshots which we obtained from our simulations are shown in Figure 1. The sequence of events happening during the collapse is captured by these snapshots. At initial time the polymer is in an extended state with fluctuations of the local monomer density along the chain. Soon there appear a number of local clusters of monomers which then start to grow by withdrawing monomers from the rest of the chain. This gives rise to the formation of the so called pearl-necklace. Once the tension in the chain is at maximum, two successive clusters along the chain coalesce with each other to grow in size. This process goes on until a single cluster or globule is formed. The final stage of the collapse is the rearrangement of the monomers within the single cluster to form a compact globule. This last stage, however, is difficult to disentangle from the previous stages.

The first two stages of formation and growth of clusters during the collapse of a polymer as demonstrated in Figure 1 are clearly reminiscent of usual coarsening phenomena in particle or spin systems. As already mentioned traditionally for studying coarsening one starts with an initial state where the distribution of particles or spins is homogeneous, e.g., homogeneous fluid or paramagnet above the critical temperature. Similarly to study the collapse kinetics one starts with a polymer in the extended coil phase which is analogous to the homogeneous phase in particle or spin systems. Usual coarsening sets in when the initial homogeneous configuration is suddenly cooled down to a temperature below the critical temperature where the equilibrium state is an ordered state, e.g., condensed droplet in fluid background or ferromagnet. Similarly, for a polymer, the collapse occurs when the temperature is suddenly brought down below the corresponding collapse transition temperature. There the equilibrium collapsed phase is analogous to the droplet phase in fluids.

Now coarsening refers to the process via which the initial homogeneous system evolves while approaching the ordered phase. This happens via the formation and subsequent growth of domains of like particles or spins. This is illustrated in the upper panel of Figure 2 where we show the time evolution of the droplet formation in a fluid starting from a homogeneous phase via MC simulations of the Ising lattice gas. At early times many small domains or droplets are formed which then coarsen to form bigger droplets and eventually giving rise to a single domain or droplet. A similar sequence of events is observed during collapse of a polymer as shown once again in the lower panel of Figure 2 which explains the 


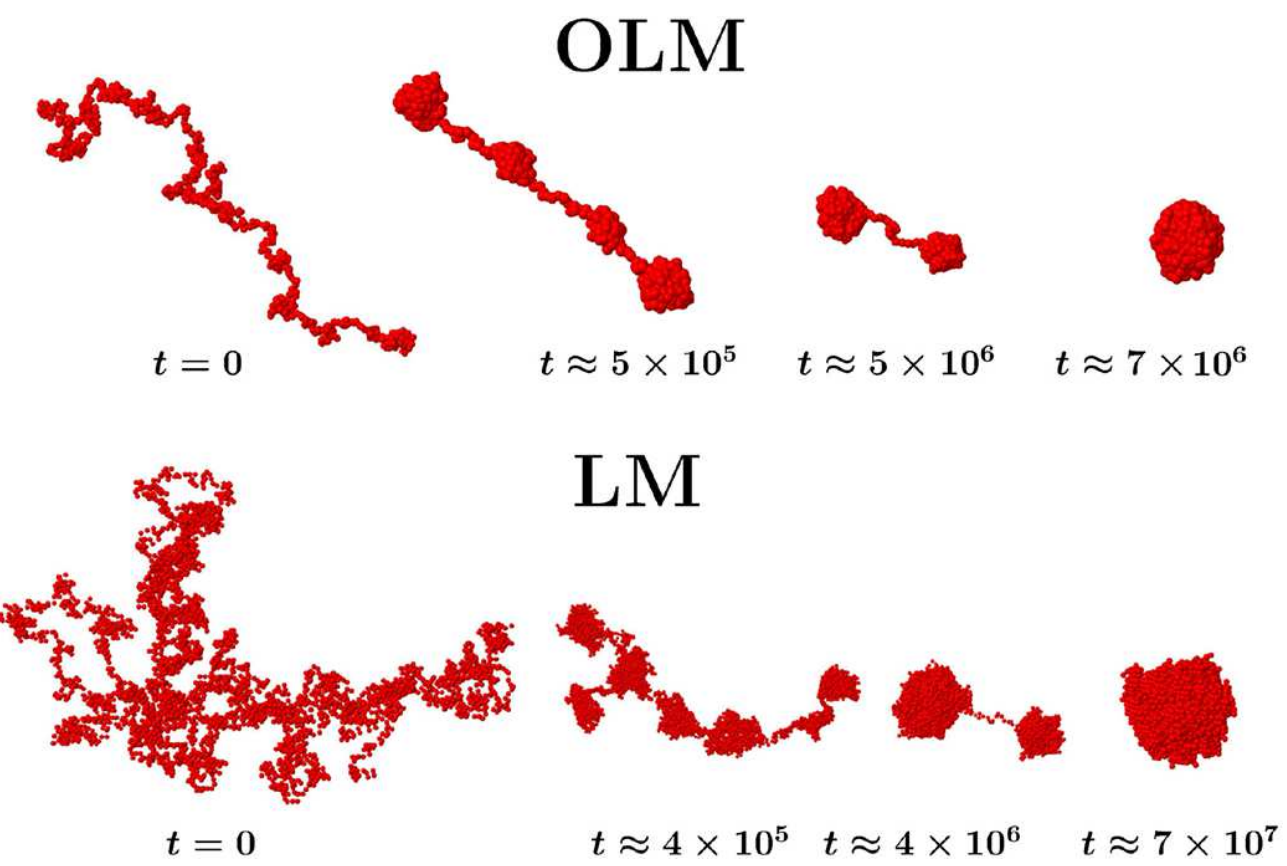

Fig. 1. Time-evolution snapshots during collapse of a homopolymer showing pearl-necklace formation, following a quench from an extended coil phase to a temperature, $T_{q}=1$ for OLM and $T_{q}=2.5$ for LM, in the globular phase. The chain lengths $N$ used are 724 and 4096 for OLM and LM, respectively. Taken from reference [41].

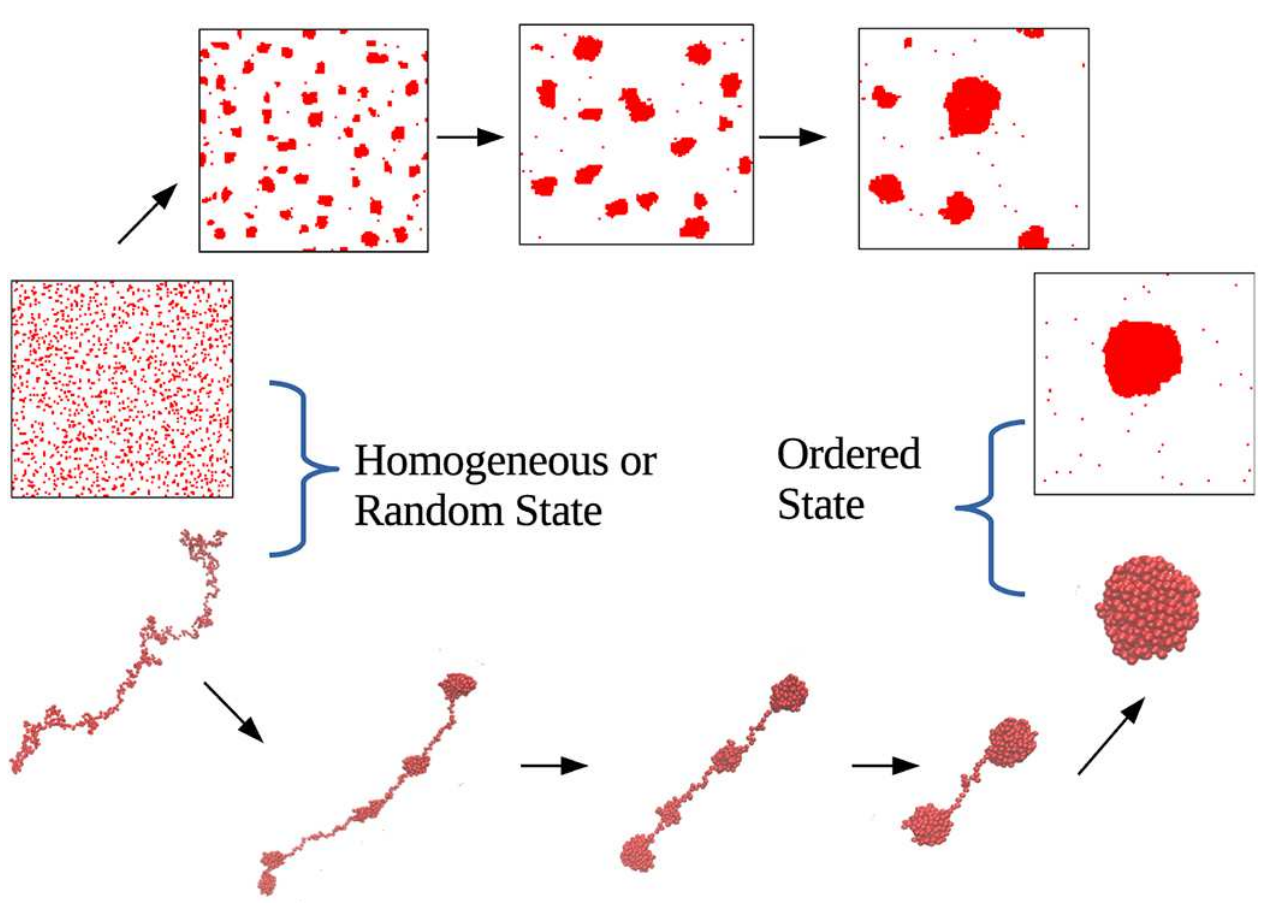

Fig. 2. Illustration of the similarities between the collapse kinetics and the usual coarsening of a particle system. The upper panel shows evolution snapshots for the droplet formation in a particle system using the Ising lattice gas in two spatial dimensions. The lower panel shows the evolution of a homopolymer obtained from simulation of the OLM. 
phenomenological analogy of collapse with usual coarsening phenomena. Coarsening from a theoretical point of view is understood as a scaling phenomenon which means that certain morphology-characterizing functions of the system at different times can be scaled onto each other using corresponding scaling functions $[4,48]$. This scaling in turn also implies that there must be scaling of the timedependent length scale, too, which in most of the cases shows a power-law scaling like in equation (2). Based on this understanding in general and the above mentioned analogy we will discuss in the remaining part of this section how to investigate the presence of nonequilibrium scaling laws in the dynamics of collapse of a homopolymer.

\subsection{Relaxation behaviour of the collapse}

In all earlier studies, the straightforward way to quantify the kinetics was to monitor the time evolution of the overall size of the polymer, i.e., the squared radius of gyration given as

$$
R_{g}^{2}=\frac{1}{N} \sum_{i=1}^{N}\left(\boldsymbol{r}_{i}-\boldsymbol{r}_{\mathrm{cm}}\right)^{2}
$$

where $\boldsymbol{r}_{\mathrm{cm}}$ is the center of mass of the polymer. In the coiled state (above $T_{\theta}$ ), $R_{g}^{2} \sim N^{2 \nu_{F}}$ with $\nu_{F}=3 / 5$, in the Flory mean-field approximation, whereas in the globular state (below $T_{\theta}$ ), $R_{g}^{2} \sim N^{2 / d}$ is much smaller [78]. Such decay of $R_{g}^{2}$ with time is shown in Figure 3a for both OLM and LM. Although in some of the earlier studies a power-law decay of $R_{g}^{2}$ is suggested, in most cases or at least in the present cases that does not work. Rather, the decay can be well described by the form

$$
R_{g}^{2}(t)=b_{0}+b_{1} \exp \left[-\left(\frac{t}{\tau_{f}}\right)^{\beta}\right] \text {, }
$$

where $b_{0}$ corresponds to the saturated value of $R_{g}^{2}(t)$ in the collapsed state, $b_{1}$ is associated with the value at $t=0$, and $\beta$ and $\tau_{f}$ are fitting parameters. For details about fitting the data with the form (13), see references [39] and [40] for OLM and LM, respectively. An illustration of how appropriately this form works is shown in Figure 3a. There the respective solid lines are fits to the form (13). While the above form does not provide any detail about the specificity of the collapse process, it gives a measure of the collapse time $\tau_{c}$ via $\tau_{f}$. However, to avoid the unreliable extraction of the collapse time from such a fitting, one could alternatively use a rather direct way of estimating $\tau_{50}$ which corresponds to the time when $R_{g}^{2}(t)$ has decayed to half of its total decay, i.e., $\left[R_{g}^{2}(0)-R_{g}^{2}(\infty)\right] / 2$. Data for both models as shown in Figure $3 \mathrm{~b}$ reflect a power-law scaling, to be quantified with the form

$$
\tau_{c}=B N^{z}+\tau_{0},
$$

where $B$ is a nontrivial constant that depends on the quench temperature $T_{q}, z$ is the corresponding dynamical
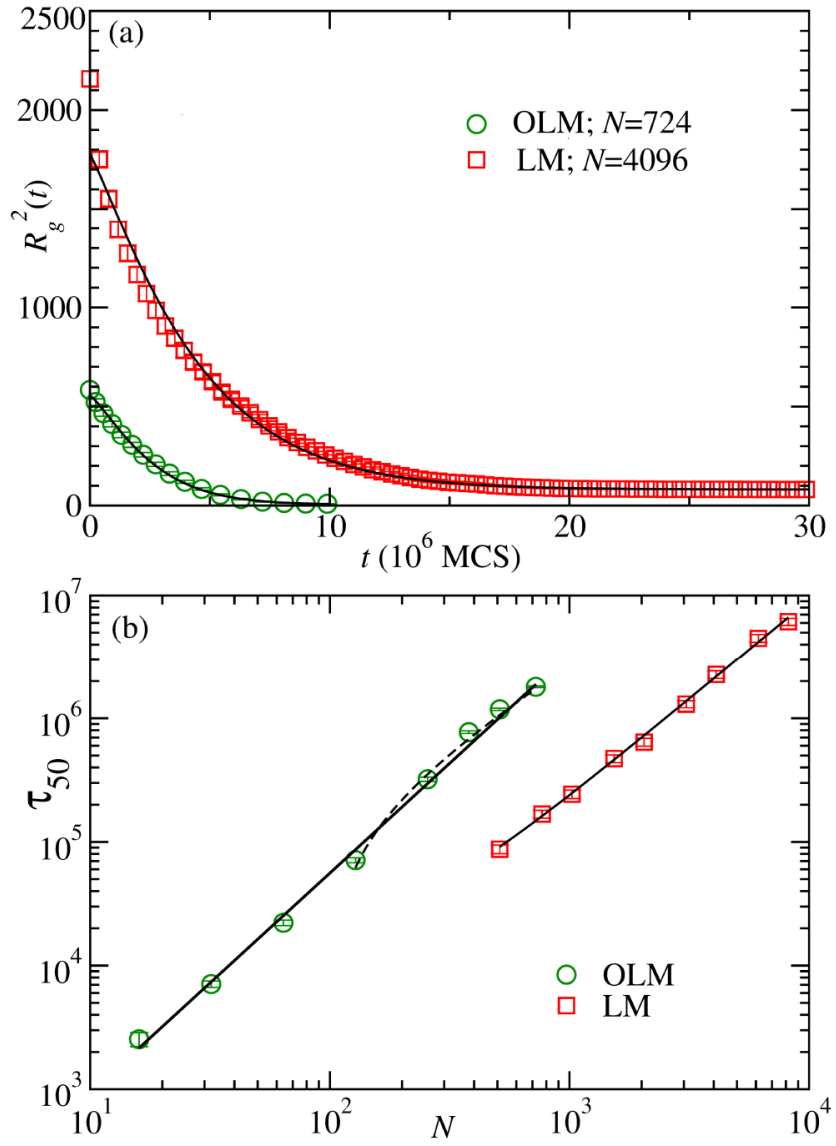

Fig. 3. (a) Time dependence of the squared radius of gyration, $R_{g}^{2}(t)$, for both OLM $\left(T_{q}=1.0\right)$ and LM $\left(T_{q}=2.5\right)$. The solid lines are fits to the data using the form described by equation (13) with $\beta=1.18$ and 1.15 for OLM and LM, respectively. (b) Scaling of the collapse time, $\tau_{50}$, with respect to $N$. The solid lines are fits to the form (14). The dashed line is a fit of the OLM data for $N \geq 128$, to the form (14) by fixing $z=1$. Adapted from reference [41].

exponent, and the offset $\tau_{0}$ comes from finite-size corrections. For LM a fitting (shown by the corresponding solid line) with the form (14) provides $z=1.61(5)$ and is almost insensitive to the chosen range. However, for OLM the fitting is sensitive to the chosen range. While using the whole range of data provides $z=1.80(6)$ (shown by the corresponding solid line), fitting only the data for $N \geq 128$ yields $z=1.20(9)$. In this regard, a linear fit [fixing $z=1$ in (14)], shown by the dashed line, also works quite well. For a comparison of the values of $z$ obtained by us with the ones obtained by others, see Table 1 .

\subsection{Coarsening during collapse}

Having the phenomenological analogy between collapse of a polymer and usual coarsening of particle and spin systems established, in this subsection we present the scaling of the cluster growth during the collapse under the light of well established protocols of the coarsening in particle or spin systems. 


\subsubsection{Scaling of morphology-characterizing functions}

Coarsening in general is a dynamical scaling phenomenon, where certain structural quantities that quantify the morphology of the system, e.g., two-point equal-time correlation functions and structure factors show scaling behaviour with time $[4,48]$. This means that the structure factors at different times can be collapsed onto the same master curve by using the relevant length scales, i.e., cluster size or domain size at those times. This fact is used to extract the relevant time-dependent length scale that governs the kinetics of coarsening. For example one uses the first moment of the structure factor at a particular time to have a measure of the length scale or the average domain size during coarsening. However, to understand the kinetics of cluster growth during the collapse of a polymer traditionally the average number of monomers present in a cluster is used as the relevant length scale $C_{s}(t)$. For studying the OLM we used this definition to calculate $C_{s}(t)$, details of which can be found in reference [39] and later will also be discussed in Section 4.1 for the $d=2$ case. The validity of this definition as the relevant length scale can be verified by looking at the expected scaling of the cluster-size distribution $P\left(C_{d}, t\right)$, i.e., the probability to find a cluster of size $C_{d}$ among all the clusters at time $t$. Using this distribution we calculate the average cluster size as $C_{s}(t)=\left\langle C_{d}\right\rangle$. The corresponding scaling behaviour is given as

$$
P\left(C_{d}, t\right) \equiv C_{s}(t)^{-1} \tilde{P}\left[C_{d} / C_{s}(t)\right]
$$

where $\tilde{P}$ is the scaling or master function. This means that when $C_{s}(t) P\left(C_{d}, t\right)$ at different times are plotted against $C_{d} / C_{s}(t)$ they should fall on top of each other. This verification is presented in Figure 4 where in the main frame we show plots of the (unscaled) distributions $P\left(C_{d}, t\right)$ at different times, and in the inset the corresponding scaling plot using the form (15). Coincidentally, here, the tail of the distribution shows an exponential decay as observed in coarsening of particle [79] and spin systems [80,81].

On the other hand, for a lattice model, one can use the advantage of having the monomers placed on lattice points. There a two-point equal-time correlation function can be defined as

$$
C(r, t)=\langle\rho(0, t) \rho(r, t)\rangle
$$

with

$$
\rho_{i}(r, t)=\frac{1}{m_{r}} \sum_{j, r_{i j}=r} \theta\left(\boldsymbol{r}_{j}, t\right)
$$

where the characteristic function $\theta$ is unity if there is a monomer at position $\boldsymbol{r}_{j}$ or zero otherwise. $m_{r}$ denotes the number of possible lattice points at distance $r$ from an arbitrary point of the lattice. Plots for such correlation functions at different times during the collapse of a polymer using LM are shown in the main frame of Figure 5. Slower decay of $C(r, t)$ as time increases suggests the presence of a growing length scale. Thus following the trend in usual coarsening studies one can extract an average

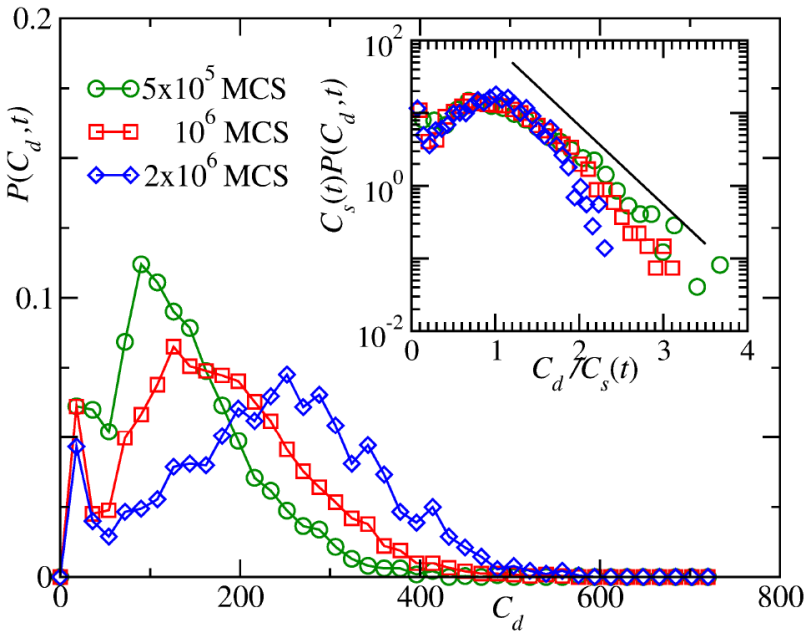

Fig. 4. Normalized distribution of the cluster sizes at three different times during the coarsening stage of the collapse at $T_{q}=1$ for a polymer with $N=724$ modeled by OLM. The inset demonstrates the scaling behaviour of the collapse phenomenon via the semi-log plot of the corresponding scaling of the distribution functions. The solid line shows consistency of the data with an exponential tail. Taken from reference [39]. (@ Royal Society of Chemistry, 2017. This figure is subject to copyright protection and is not covered by a Creative Commons license.)

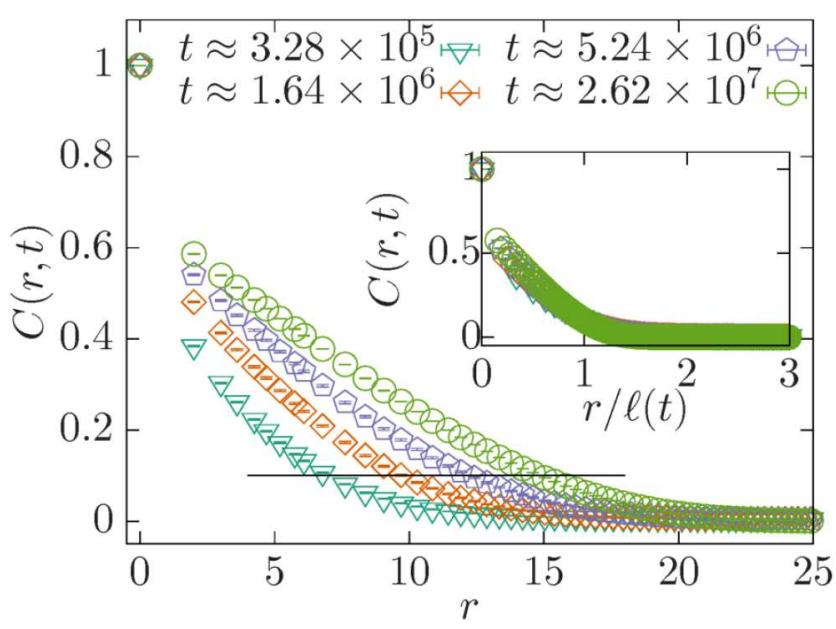

Fig. 5. Morphology characterizing two-point equal-time correlation function $C(r, t)$ at different times, showing the presence of a growing length scale during collapse of a polymer obtained via simulation of LM with $T_{q}=2.5$ and $N=4096$. The inset shows the presence of scaling in the process via a plot of the same data as a function of $r / \ell(t)$ where $\ell(t)$ is the characteristic length scale calculated using (18) with $h=0.1$. Adapted from reference [40]. (@ AIP, 2017. This figure is subject to copyright protection and is not covered by a Creative Commons license.)

length scale $\ell(t)$ that characterizes the clustering during the collapse, via the criterion

$$
C(r=\ell(t), t)=h,
$$


where $h$ denotes an arbitrary but reasonably chosen value from the decay of $C(r, t)$. Calculation of $\ell(t)$ in the above manner automatically suggests to look for the dynamical scaling of the form

$$
C(r, t) \equiv \tilde{C}(r / \ell(t))
$$

where $\tilde{C}$ is the scaling function. Such a scaling behaviour is nicely demonstrated in the inset of Figure 5, where we show the corresponding data presented in the main frame as function of $r / \ell(t)$. Note that here $\ell(t)$ gives the linear size of the ordering clusters. Thus in order to compare $\ell(t)$ of LM with the cluster size $C_{s}(t)$ obtained for OLM one must use the relation $\ell(t)^{d} \equiv C_{s}(t)$. For a check of the validity of this relation, see reference [40].

\subsubsection{Cluster growth}

Once it is established that the coarsening stage of polymer collapse is indeed a scaling phenomenon, the next interest goes towards checking the associated growth laws. In Figure 6a, we show the time dependence of $C_{s}(t)$ for OLM and LM. To make the data from both models visible on the same scale there the $y$-axis is scaled by the corresponding chain length $N$ of the polymer. Note that saturation of the data for LM at a value less than unity is due to the fact that there we have calculated the average cluster size $C_{s}(t)$ from the decay of the correlation function $C(r, t)$ as described in the previous subsection. This gives a proportionate measure of the average number of monomers present in the clusters and thus the data saturate to a value less than unity.

In coarsening kinetics of binary mixtures such time dependence of the relevant length scale can be described correctly when one considers an off-set in the scaling ansatz $[80,82-84]$. Similarly, it was later proved to be appropriate for the cluster growth during the collapse of a polymer $[36,39]$. Following this one writes down the scaling ansatz as

$$
C_{s}(t)=C_{0}+A t^{\alpha_{c}},
$$

where $C_{0}$ corresponds to the cluster size after crossing over from the initial cluster formation stage, and $A$ is a temperature-dependent amplitude. The solid lines in Figure $6 \mathrm{a}$ are fits to the form (20) yielding $\alpha_{c}=0.98(4)$ and $0.62(5)$ for OLM and LM, respectively.

One can verify the robustness of the growth by studying the dependence of cluster growth on the quench temperature $T_{q}$. For this one uses data at different $T_{q}$ and can perform a scaling analysis based on nonequilibrium finitesize scaling (FSS) arguments [39]. The nonequilibrium FSS analysis was constructed based on FSS analyses in the context of equilibrium critical phenomena $[85,86]$. An account of the FSS formulation in the present context can be found in reference [39]. In brief, one introduces in the growth ansatz (20) a scaling function $Y\left(y_{p}\right)$ as

$$
C_{s}(t)-C_{0}=\left(C_{\max }-C_{0}\right) Y\left(y_{p}\right),
$$
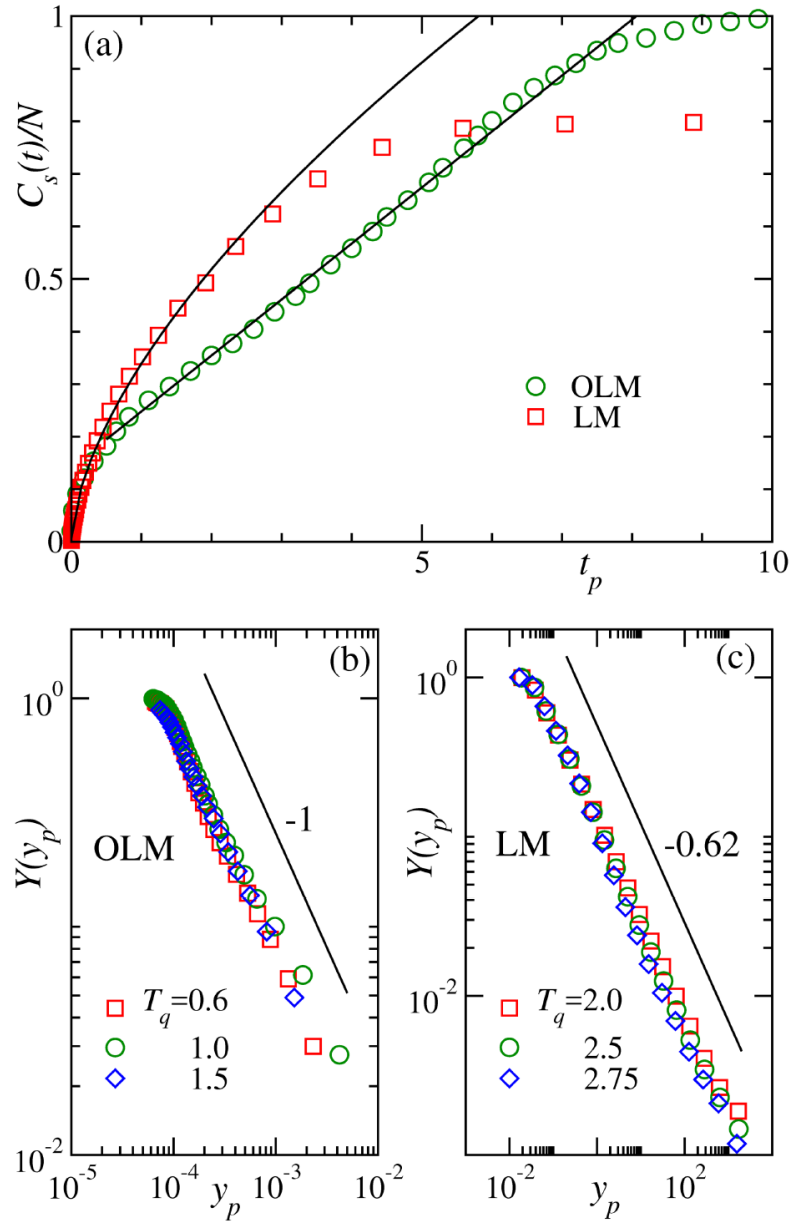

Fig. 6. (a) Plots of the average cluster size $C_{s}(t) / N$, as function of time for the systems presented in Figure 1. To make both the data visible on the same plot, we divide the time axis by a factor $m$ to obtain $t_{p}=t / m$, where $m=1 \times 10^{6}$ and $3.5 \times 10^{6}$ for OLM and LM, respectively. The solid lines are fits to the form (20) with $\alpha_{c}=0.98$ for OLM and $\alpha_{c}=0.62$ for LM. The plots in (b) and (c) demonstrate the scaling exercise for OLM with $\alpha_{c}=1.0$ and LM with $\alpha_{c}=0.62$, respectively, showing that data for $C_{s}(t)$ at different quench temperatures $T_{q}$ can be collapsed onto a master curve using a nonuniversal metric factor in the scaling variable. The solid lines represent the corresponding $Y\left(y_{p}\right) \sim y_{p}^{-\alpha_{c}}$ behaviour. Adapted from reference [41].

which implies

$$
Y\left(y_{p}\right)=\frac{C_{s}(t)-C_{0}}{C_{\max }-C_{0}}
$$

where $C_{\max } \sim N$ is the maximum cluster size a finite system can attain. In order to account for the temperaturedependent amplitude $A\left(T_{q}\right)$, one uses the scaling variable

$$
y_{p}=f_{s} \frac{\left(N-C_{0}\right)^{1 / \alpha_{c}}}{t-t_{0}}
$$


where

$$
f_{s}=\left[\frac{A\left(T_{q, 0}\right)}{A\left(T_{q}\right)}\right]^{1 / \alpha_{c}}
$$

The metric factor $f_{s}$ is introduced for adjusting the nonuniversal amplitudes $A\left(T_{q}\right)$ at different $T_{q}$. Here, in addition to $C_{0}$ one also uses the crossover time $t_{0}$ from the initial cluster formation stage. A discussion of the estimation of $C_{0}$ and $t_{0}$ can be found in references $[39,40]$. While performing the exercise we tune the parameters $\alpha_{c}$ and $f_{s}$ to obtain a data collapse along with the $Y\left(y_{p}\right)$ $\sim y_{p}^{-\alpha_{c}}$ behaviour in the finite-size unaffected region. In Figures $6 \mathrm{~b}$ and $6 \mathrm{c}$, we demonstrate such scaling exercises with $\alpha_{c}=1.0$ and 0.62 for OLM and LM, respectively. For $f_{s}$, we use the reference temperature $T_{q, 0}=1.0$ and 2.0 for OLM and LM, respectively. The collapse of data for different $T_{q}$ and consistency with the corresponding $y_{p}^{-\alpha_{c}}$ behaviour in both plots suggest that the growth is indeed quite robust and can be described by a single universal FSS function with nonuniversal metric factor $f_{s}$ in the scaling variable. However, $\alpha_{c}$ in OLM is larger than for LM, a fact in concurrence with the values of $z$ estimated previously, and thus to some extent providing support to the heuristic relation $z \sim 1 / \alpha_{c}$. The use of a nonuniversal metric factor in order to find a universal FSS function was first introduced in the context of equilibrium critical phenomena using different lattice types [87,88]. After adapting this concept to nonequilibrium FSS of polymer kinetics in references $[39,40]$ as explained above, it was recently also transferred to spin systems where its usefulness has been demonstrated in a coarsening study of the Potts model with conserved dynamics [81].

\subsection{Aging and related scaling}

Apart from the scaling of the growing length scale or the cluster size that deals only with equal-time quantities, coarsening processes are associated with the aging phenomenon as well. Thus along the same line, in order to check aging during collapse of a polymer one can calculate the two-time correlation or autocorrelation function described in equation (4). However, unlike for spin systems here the choice of the observable $O_{i}$ is not trivial. Nevertheless, for OLM we identified the observable $O_{i}$ as a variable based on the cluster identification method. We assign $O_{i}= \pm 1$ depending on whether the monomer is inside $(+1)$ or outside $(-1)$ a cluster. It is apparent that our cluster identification method is based on the local density around a monomer along the chain. Thus $C\left(t, t_{w}\right)$ calculated using this framework gives an analogue of the usual density-density autocorrelation function in particle systems. On the other hand for LM, we assign $O_{i}= \pm 1$ by checking the radius $r$ at which the local density, given by $\rho_{i}(r, t)$ [see Eqs. (16) and (17)], first falls below a threshold of 0.1 . If this radius is smaller than $\sqrt{3}$ we assign $O_{i}=1$, marking a high local density, otherwise we chose $O_{i}=-1$ to mark a low local density. For details see references [39] and [40] for OLM and LM, respectively.
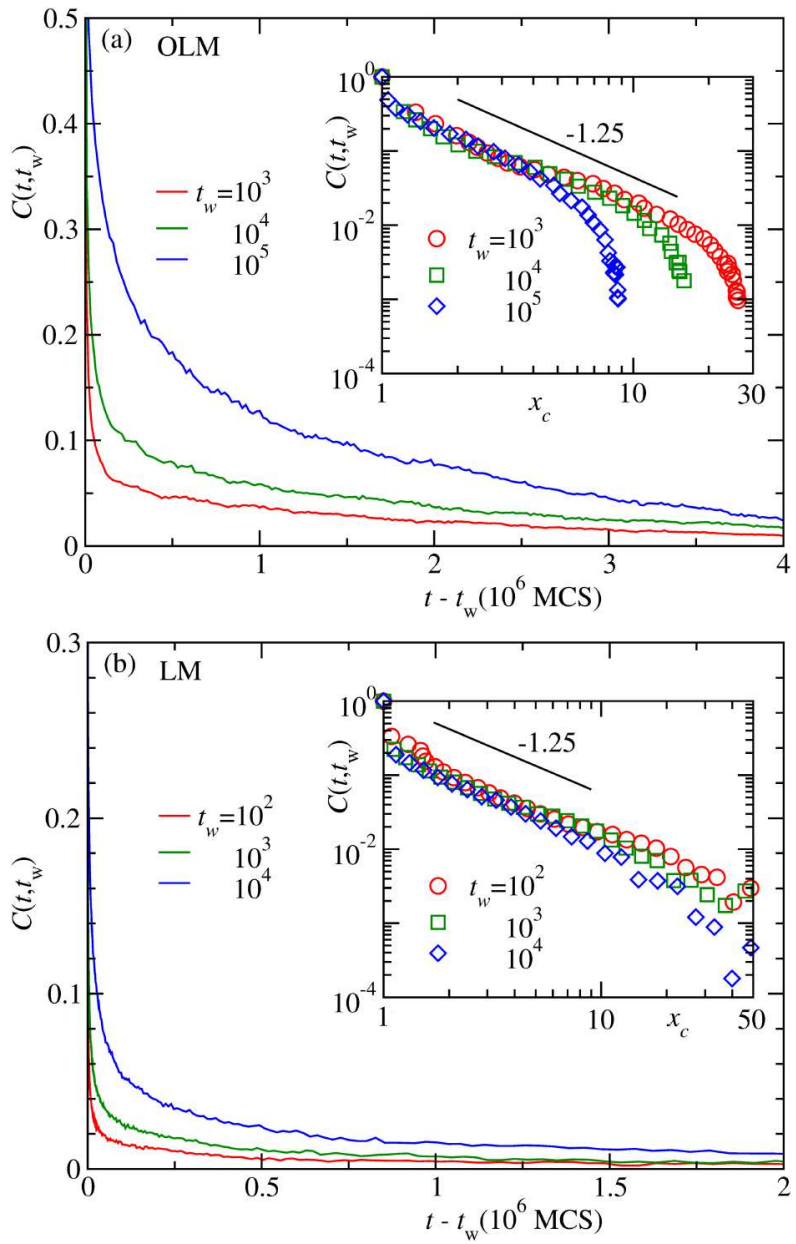

Fig. 7. Demonstration of aging phenomenon during collapse of a polymer for (a) OLM $\left(N=724, T_{q}=1.0\right)$ and (b) LM $\left(N=8192, T_{q}=1.5\right)$. The main frames show plots of the autocorrelation functions calculated using (4) at different waiting times $t_{w}$, as mentioned there. The insets show the corresponding scaling plots with respect to the scaling variable $x_{c}=C_{s}(t) / C_{s}\left(t_{w}\right)$, in accordance with (7). The solid lines depict the consistency of the data with a power law having an exponent $\lambda_{c}=1.25$. The plots in (a) and (b) are adapted from references [39] and [40], respectively. (Panels (a) and (b) are subject to copyright protection and are not covered by a Creative Commons license.)

In the main frames of Figures $7 \mathrm{a}$ and $7 \mathrm{~b}$ we show plots of the autocorrelation function $C\left(t, t_{w}\right)$ against the translated time $t-t_{w}$ for (a) OLM and (b) LM. Data from both the cases clearly show breaking of time-translation invariance, one of the necessary conditions for aging. It is also evident that as $t_{w}$ increases, the curves decay more slowly, an indication of slow relaxation behaviour fulfilling the second necessary condition for aging. As a check of the final condition for aging, i.e., dynamical scaling, in principle one could study the scaling with respect to the scaled time $t / t_{w}$. Although such an exercise provides a reasonable collapse of data for OLM, data for LM do not show scaling with respect to $t / t_{w}$. In this regard, one could look for special aging behaviour that can be achieved by 
considering [58]

$$
C\left(t, t_{w}\right) \equiv G\left(\frac{h(t)}{h\left(t_{w}\right)}\right)
$$

with the scaling variable

$$
h(t)=\exp \left(\frac{t^{1-\mu}-1}{1-\mu}\right) .
$$

Here, $G$ is the scaling function and $\mu$ is a nontrivial exponent. Special aging with $0<\mu<1$ is referred to as subaging and has been observed mostly in soft-matter systems [69-71], in spin glasses [89-91], and recently in long-range interacting systems [92]. The $\mu>1$ case is referred to as superaging and was claimed to be observed in site-diluted Ising ferromagnets. However, Kurchan's lemma [93] rules out the presence of apparent superaging [94]. This was further consolidated via numerical evidence in reference [95]. There it has been argued that the true scaling is observed in terms of the ratio of growing length scales at the corresponding times, i.e., $\ell(t) / \ell\left(t_{w}\right)$. In the case of polymer collapse with LM, too, one apparently observes special scaling of the form (25) with $\mu<1$, i.e., subaging in this case. However, following the argument of Park and Pleimling [95], one gets also here the simple scaling behaviour with respect to the scaling variable $x_{c}=C_{s}(t) / C_{s}\left(t_{w}\right)$, thus ruling out the presence of subaging. Such scaling plots of the autocorrelation data both for OLM and LM are shown in the insets of Figure 7. In both cases the data seem to follow the power-law scaling with a decay exponent $\lambda_{c} \approx 1.25$.

Relying on the fact that the calculation of $C\left(t, t_{w}\right)$ is based on the cluster identification criterion, i.e., by calculating the local monomer densities around each monomer along the polymer chain, it gives an analogue to the usual density-density autocorrelation function as used in glassy systems. Keeping in mind the corresponding argument for the bounds on the respective autocorrelation exponent for spin-glass and ferromagnetic ordering, one can thus assume [37] $C\left(t, t_{w}\right) \sim\left\langle\rho(t) \rho\left(t_{w}\right)\right\rangle$ where $\rho$ is the average local density of monomers. Now let us consider a set of $C_{s}$ monomers at $t\left(\gg t_{w}\right)$ and assume that at $t_{w}$ the polymer is more or less in an extended coil state where the squared radius of gyration scales as $R_{g}^{2} \sim N^{2 \nu_{F}}$. Using $C_{s} \equiv N$ in this case one can write

$$
\rho\left(t_{w}\right) \sim C_{s} / R_{g}{ }^{d} \sim C_{s}^{-\left(\nu_{F} d-1\right)} .
$$

The above fact can be verified from Figures $8 \mathrm{a}$ and $8 \mathrm{~b}$ for OLM and LM, respectively, where we plot the average geometrical (Euclidean) distance $R_{e}\left(\sim R_{g}\right)$ between the monomers $i$ and $j$ placed at a distance $|i-j|$ along the contour of the chain at different times during the collapse. For both cases, the data at early times show that the behaviour is consistent with an extended coil governed by the Flory exponent $\nu_{F}=3 / 5$. This consolidates the foundation of the relation (27) provided $t_{w}$ is at early times.
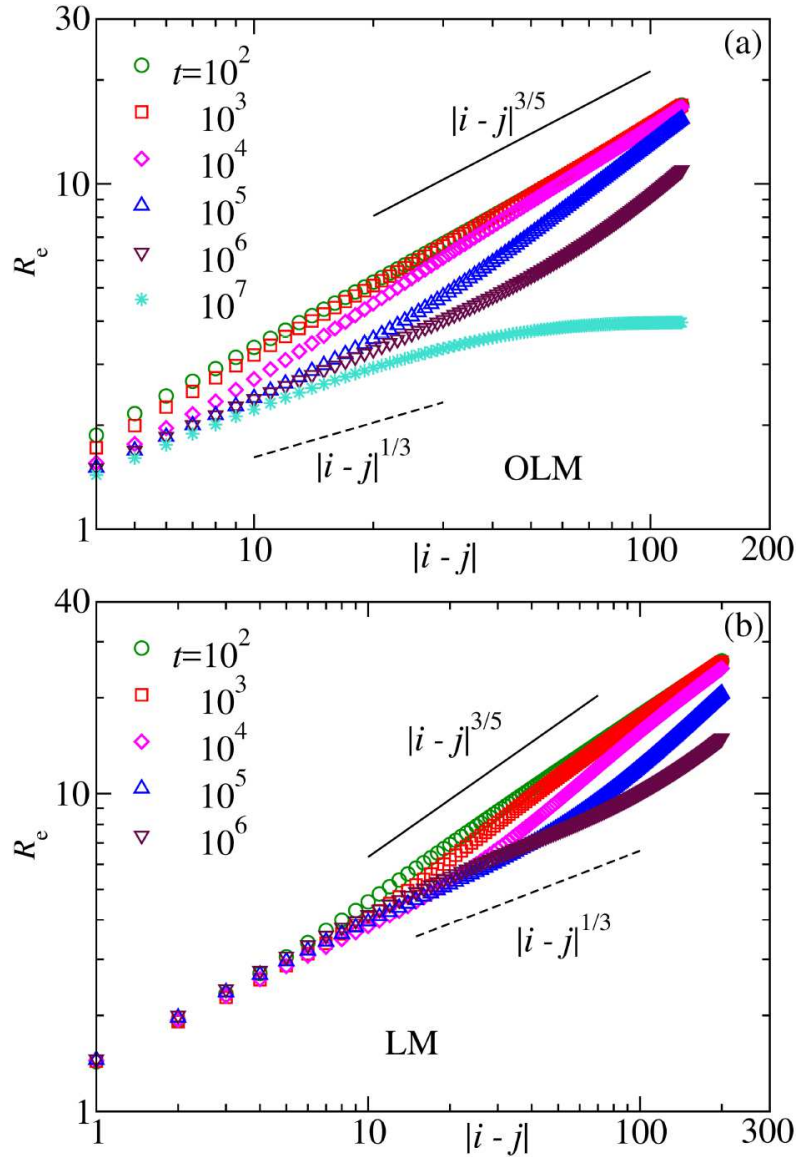

Fig. 8. Geometrical distance between monomers $i$ and $j$ which are at a distance $|i-j|$ along the contour of the chain for a polymer using (a) OLM and (b) LM, at different times mentioned. The respective chain lengths are $N=724$ and 2048 and the quench temperatures are $T_{q}=1.0$ and 2.5. The solid line shows the expected behaviour for an extended coil and the dashed line shows the behaviour in the collapsed phase. The plot in (a) is taken from reference [39]. (Panel (a) is subject to copyright protection and is not covered by a Creative Commons license.)

Now at the observation time $t$ there are two possibilities. Firstly, if $t$ is late enough, then we expect that all the monomers will be inside a single cluster which gives $R_{g} \sim$ $C_{s}^{1 / d}$ so that $\rho(t)=1$. Thus considering the maximum overlap between $\rho(t)$ and $\rho\left(t_{w}\right)$ we get

$$
C\left(t, t_{w}\right) \sim C_{s}^{-\left(\nu_{F} d-1\right)} .
$$

This gives the lower bound. Secondly, with the assumption that the polymer is in an extended coil state even at time $t$, then $\rho(t)=\rho\left(t_{w}\right)$ holds and we obtain

$$
C\left(t, t_{w}\right) \sim C_{s}^{-2\left(\nu_{F} d-1\right)},
$$

providing the upper bound for the autocorrelation exponent $\lambda_{c}$. Thus by combining (28) and (29) we arrive at the 
bounds [37]

$$
\left(\nu_{F} d-1\right) \leq \lambda_{c} \leq 2\left(\nu_{F} d-1\right) .
$$

Putting $\nu_{F}=3 / 5$ in (30) one would get $4 / 5 \leq \lambda_{c} \leq 8 / 5$. Further, inserting the more precise numerical estimate in $d=3$ as $[96,97] \nu_{F}=0.587597$, we get

$$
0.762791 \leq \lambda_{c} \leq 1.525582 .
$$

The validity of this bound can also be readily verified from the consistency of our data in the insets of Figure 7 with the solid lines having a power-law decay with exponent 1.25. We make the choice of $t_{w}$ in all the plots so that the assumption that at time $t_{w}$ the polymer is in an extended coil state is valid. This choice can also be appreciated from the plots in Figures 8a and 8b for OLM and LM, respectively. There it is evident that the extended coil behaviour $\left(R_{e} \sim|i-j|^{3 / 5}\right)$ at early times is gradually changing to the behaviour expected for the collapsed phase $\left(R_{e} \sim|i-j|^{1 / d}\right.$ with $\left.d=3\right)$ at late times. The little off behaviour of the data for higher $t_{w}$ in the inset of Figure 7 is indeed due to the fact that at those times the formation of stable clusters has already initiated to change the extended coil behaviour of the chain. Confirmation of the value of $\lambda_{c}$ via FSS can also be done as presented in references $[37,40]$.

To confirm the robustness of the above bound and the value of $\lambda_{c}$, we plot $C\left(t, t_{w}\right)$ from different temperatures $T_{q}$ in Figure 9a for OLM and Figure $9 \mathrm{~b}$ for LM. Mere plotting of those data yields curves that are parallel to each other due to different amplitudes. However, if one uses a multiplier $f$ on the $y$-axis to adjust those different amplitudes for different $T_{q}$ one obtains curves that fall on top of each other as shown. The values of $f$ used for different $T_{q}$ are mentioned in the tables within the plots. Note that this non-trivial factor $f$ is similar to the nonuniversal metric factor $f_{s}$ used for the cluster growth in the previous subsection. The solid lines in both the cases show the consistency of the data with the scaling form (7) with $\lambda_{c}=1.25$. To further check the universality of the exponent $\lambda_{c}$ we now compare the results from aging scaling obtained for the polymer collapse using the two polymer models. For that we plot in Figure 9c the data for different $T_{q}$ coming from both models on the same graph. Here again, we have used the multiplier $f$ for the data collapse. Collapse of data irrespective of the model and the temperatures $T_{q}$ onto a master-curve behaviour and their consistency with the power-law scaling (7) having $\lambda_{c}=1.25$ (shown by the solid line), speaks for the universal nature of aging scaling during collapse of a polymer.

\section{Results for the case of OLM in $d=2$}

In this section we present some preliminary results for the kinetics of polymer collapse in $d=2$ dimensions using only OLM as defined by equations (8), (9), and (10). Experiments on polymer dynamics are often set up by using an attractive surface which effectively confines the polymer to
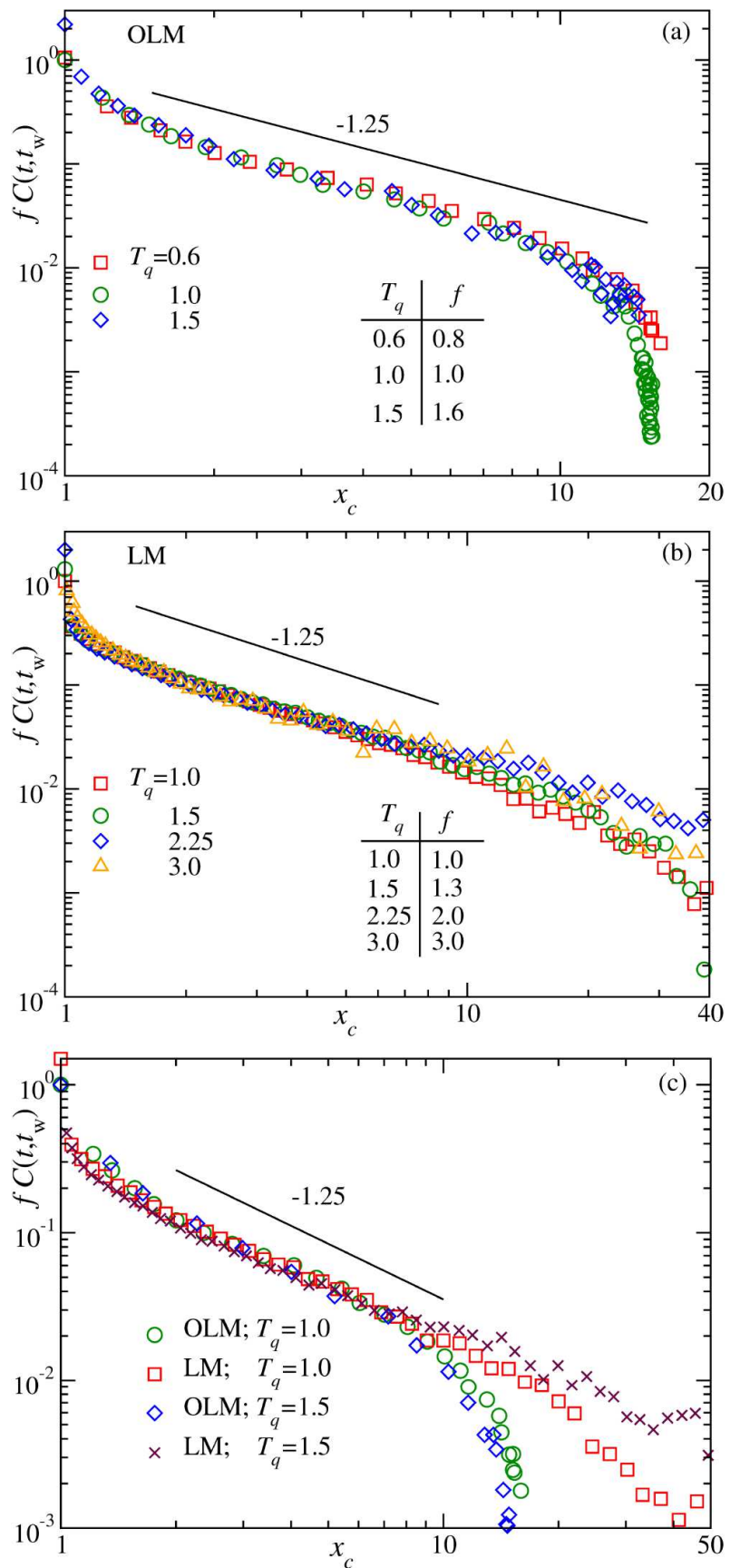

Fig. 9. Plots demonstrating that aging scaling of the autocorrelation function $C\left(t, t_{w}\right)$ at different $T_{q}$ for (a) OLM and (b) LM can be described by a single master curve when plotted as a function of $x_{c}=C_{s}(t) / C_{s}\left(t_{w}\right)$. The solid lines there again correspond to (7) with $\lambda_{c}=1.25$. For OLM, the used data are at $t_{w}=5 \times 10^{3}, 10^{4}$ and $3 \times 10^{4}$, respectively, for $T_{q}=0.6,1.0$ and 1.5. For LM, data for all temperatures are at $t_{w} \approx 10^{3}$. Note that here we have simply multiplied the $y$ axis by a factor $f$ to make the data fall onto the same master curve. (c) Illustration of the universal nature of aging scaling in the two models. Here the used data are at $t_{w}=10^{4}$ and $10^{3}$ for OLM and LM, respectively. In all the plots $N=724$ and 4096 for OLM and LM, respectively. The plot in (a) is taken from reference [39], and (b) and (c) are adapted from references $[40,41]$. (This figure is subject to copyright protection and is not covered by a Creative Commons license.) 

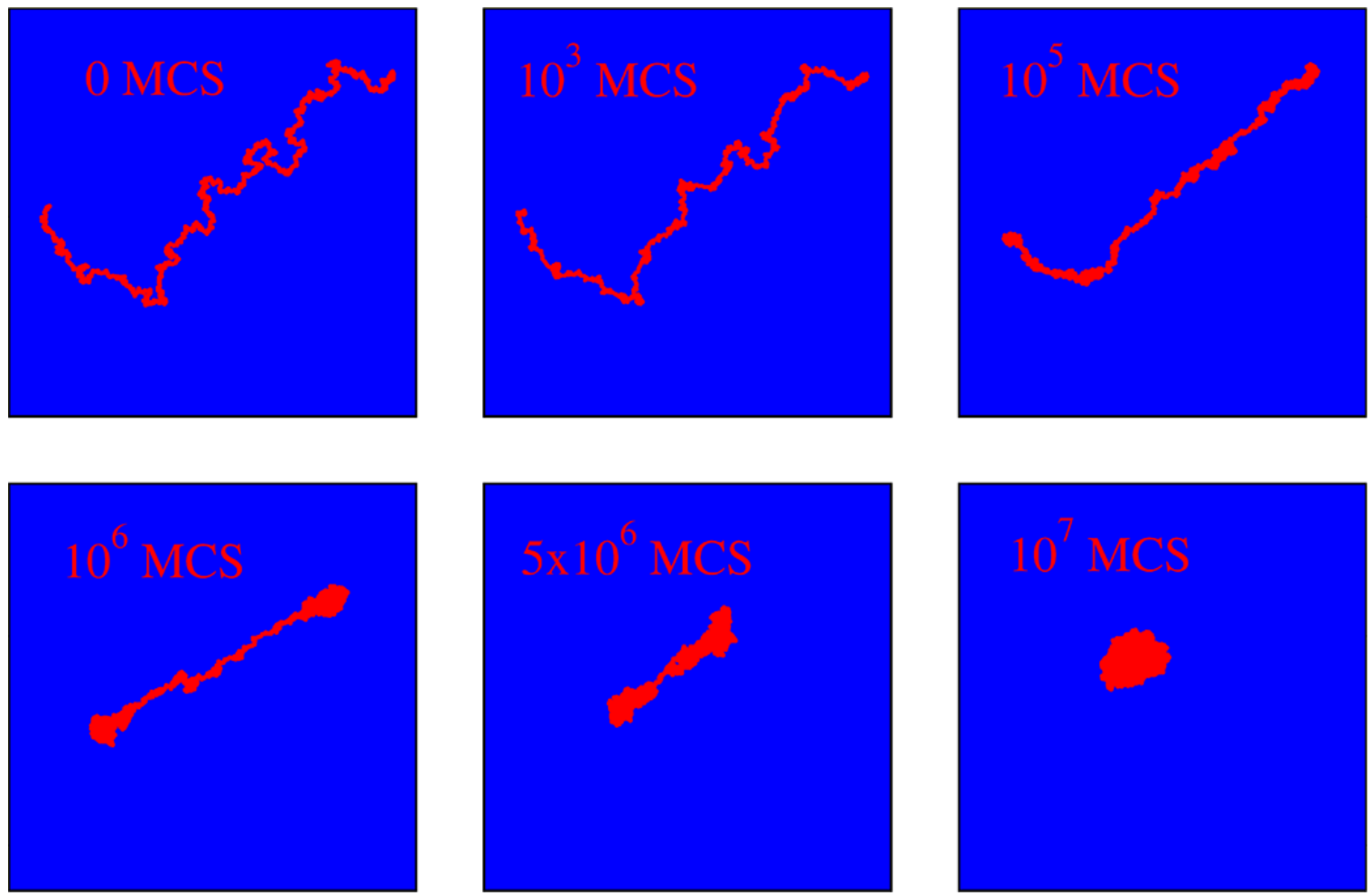

Fig. 10. Plot showing the time evolution of a polymer in $d=2$ using OLM after being quenched from a high-temperature extended coil phase to a temperature $T_{q}=1.0$ where the equilibrium phase is globular. The times are mentioned in there and the used chain length is $N=512$.

move in two-dimensional space. Thus understanding the scenario in pure $d=2$ dimensions provides some impression about such quasi-two-dimensional geometry $[5,98]$. From a technical point of view, simple Metropolis simulations of a polymer in $d=2$ are much more time consuming than in $d=3$. This is due to the absence of one degree of freedom which makes the collapse of the polymer difficult via local moves and thereby increasing the intrinsic time scale of collapse. In fact even in equilibrium there are very few studies [99-102] and in particular we did not find any study that gives an idea about the collapse transition temperature. Since for the study of the kinetics the actual value of the transition temperature is not crucial we performed a few equilibrium simulations in $d=2$ covering a wide range of temperatures and found that at $T_{q}=1.0$ the polymer is in the collapsed phase for a chain length of $N=512$, while it remains in an extended coil state at $T_{h}=10.0$. So for this work we have used a polymer of length $N=512$ and prepared an initial configuration at $T_{h}=10.0$ before quenching it to a temperature $T_{q}=1.0$. All the other specifications for the simulation method remain the same as we discussed it for OLM in Section 3.1, apart from confining the displacement moves to only $d=2$ dimensions.

In Figure 10 we show the time evolutions during the collapse of the $d=2$ polymer at $T_{q}=1.0$. The sequence of events portrayed by the snapshots shows formation of local ordering as observed for $d=3$, although the formation of a "pearl-necklace" is not so evident. By comparing with the snapshots presented for $d=3$ in

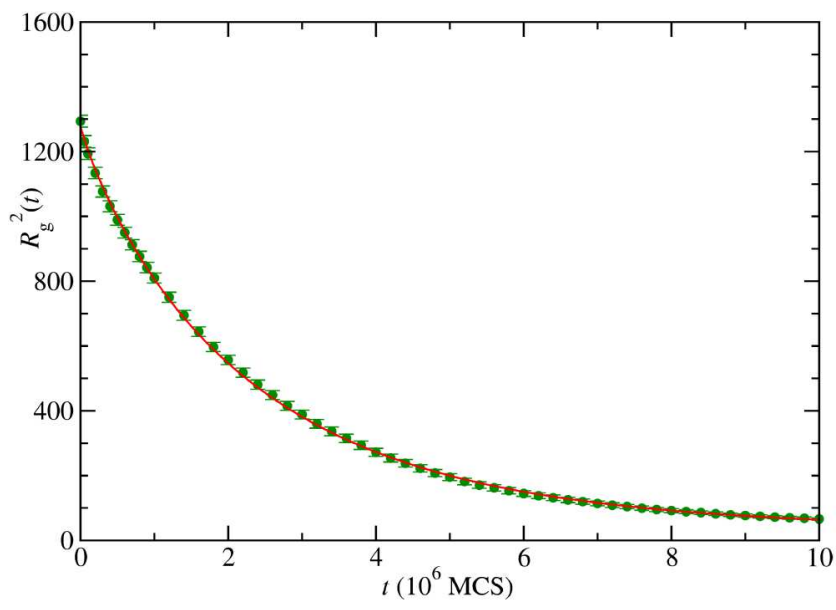

Fig. 11. Time dependence of the average squared radius of gyration $R_{g}^{2}$ during collapse of a polymer in $d=2$. The system size and the quench temperature are the same as in Figure 10. The continuous line is a fit to the data using equation (13).

Figures 1 and 2, it is apparent that the initial process of local cluster formation is much slower in $d=2$. However, once the local clusters have been formed (as shown in the snapshot at $t=10^{6} \mathrm{MCS}$ ) the time evolution shows coarsening of these clusters to finally form a single cluster or globule. Thus the overall phenomenology seems to be in line with the $d=3$ case. 
Following what has been done for the $d=3$ case, at first we look at the time dependence of the overall size of the polymer by monitoring the squared radius of gyration $R_{g}^{2}$. In Figure 11 we show the corresponding plot of $R_{g}^{2}$ (calculated as an average over 300 different initial realizations). Like in the $d=3$ case, the decay of $R_{g}^{2}$ can be described quite well via the empirical relation mentioned in equation (13). The best fit obtained is plotted as a continuous line in the plot. The obtained value of the non-trivial parameter $\beta$ in this fitting is $\approx 0.89$, which is compatible with the $d=3$ case [39]. Still, the dependence of $\beta$ on the chain length $N$ would be worth investigating and will be presented elsewhere. Along the same line an understanding of the scaling of the collapse time with the chain length will be interesting to compare with the $d=3$ case. As this Colloquium is focused more on the cluster coarsening and aging during the collapse, here, we abstain ourselves from presenting results concerning the scaling of the collapse time.

\subsection{Cluster coarsening in $d=2$}

As can be seen from the snapshots in Figure 10, during the course of the collapse, like in $d=3$, also for $d=2$ one notices formation of local clusters which via coalescence with each other merge into bigger clusters and eventually form a single cluster or globule. We measure the average cluster size in the following way. First we calculate the total numbers of monomers in the nearest vicinity of the $i$ th monomer as

$$
n_{i}=\sum_{j=1}^{N} \Theta\left(r_{c}-r_{i j}\right),
$$

where $r_{c}$ is the cutoff distance used in the nonbonded energy (9) and $\Theta$ is the Heaviside step function. If $n_{i} \geq$ $n_{\min }$, where $n_{\min }$ is a tunable lower cutoff, this is interpreted as signal for a cluster around the $i$ th monomer that contains at least those $n_{i}$ closeby monomers. Note that by construction we treat agglomerates of less than $n_{\min }$ monomers as random fluctuation or noise and do not classify them as a cluster. By performing this construction for all $N$ monomers, one obtains the number of monomers that are inside a cluster. This number, however, would greatly overestimate the total number of clusters since neighbouring monomers typically signal one and the same cluster. We remove this overcounting by considering the associated Venn diagram, that is for each monomer with $n_{i} \geq n_{\text {min }}$ we associate a set $A_{i}$ containing the $n_{i}$ neighbouring monomers satisfying the $\Theta$-constraint in (32) and then check the intersection of different sets $A_{i}$ and $A_{j}$. If the intersection is the empty set, the clusters associated with monomers $i$ and $j$ are different, otherwise the $i$ th and $j$ th monomers belong to the same cluster formed by the union of $A_{i}$ and $A_{j}$ (and hence consisting of more than $\max \left\{n_{i}, n_{j}\right\}$ monomers). The resulting set $A_{i} \cup A_{j}$ obtained this way could again be intersecting with another set which can be tackled in the same way. Thus we do this exercise repeatedly until we get a number of discrete sets that correspond to the discrete clusters.
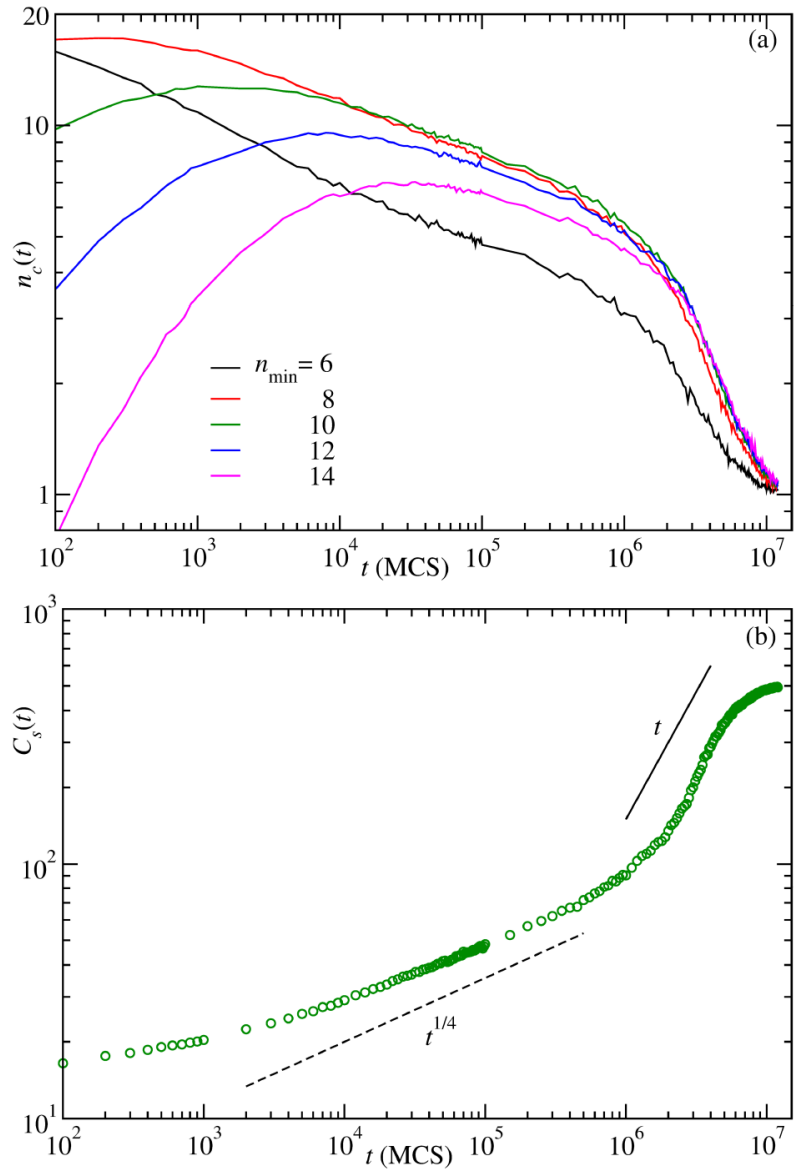

Fig. 12. (a) Plot of the average number of clusters of monomers $n_{c}$ as a function of time during collapse of a polymer with chain length $N=512$ modeled via OLM in $d=2$ at $T_{q}=1.0$. Results for different choices of $n_{\text {min }}$ are shown demonstrating the late-time consistency of the data with each other. (b) Illustration of the scaling of the cluster growth during collapse via a plot of the average cluster size $C_{s}$ as a function of time. Here we have used $n_{\text {min }}=12$. The dashed and the solid lines correspond to different power-law behaviours observed at early and late times, respectively.

In this way the actual number $n_{c}(t)$ of discrete clusters $k=1, \ldots, n_{c}(t)$ can be identified and the number of monomers $m_{k}$ within each cluster determined. Finally the average cluster size follows as

$$
C_{s}(t)=\frac{1}{n_{c}(t)} \sum_{k=1}^{n_{c}(t)} m_{k} .
$$

Note that in this calculation we do not vary the cutoff radius $r_{c}$ and fix it to the same value $\left(r_{c}=2.5 \sigma\right)$ as we have used for our simulations. Hence, the obtained values of $n_{c}(t)$ and $C_{s}(t)$ depend only on one nontrivial parameter, namely $n_{\min }$. Figure 12a shows how the above cluster identification depends on different choices for $n_{\min }$ during the collapse of a polymer having length $N=512$. There we have plotted the average number of clusters as a function of time for different $n_{\min }$. One can notice for choices 


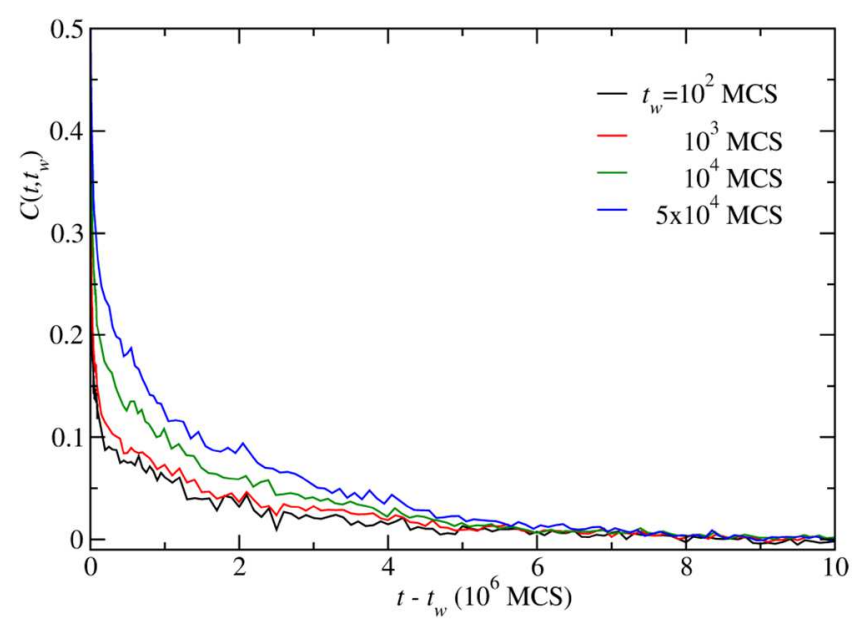

Fig. 13. Demonstration of the breakdown of time-translation invariance by plotting the autocorrelation function $C\left(t, t_{w}\right)$ as a function of the translated time $t-t_{w}$, during collapse of a polymer in $d=2$ modeled by the OLM. The chain length and $T_{q}$ are the same as in Figure 12. The chosen values of the waiting times $t_{w}$ are mentioned within the graph.

of $n_{\min } \geq 10$ the late-time behaviours are more or less indistinguishable. However, the initial structure formation stage is well covered by the choice $n_{\min }=12$. Thus we consider $n_{\min }=12$ as the optimal value to identify and calculate the average cluster size.

In Figure 12b we show the time dependence of the average cluster size. One can clearly see the presence of two distinct phases. The early-time phase corresponds to the stage of stable cluster formation ( $\left.\leq 10^{6} \mathrm{MCS}\right)$ and the later phase is the coarsening phase. The early-time data are consistent with a behaviour $C_{s}(t) \sim t^{1 / 4}$ which is slower than the corresponding behaviour in $d=3$ (see Fig. 8(b) in Ref. [39]). At late times, on the other hand, the data can be described by a $C_{s}(t) \sim t$ behaviour consistent with a $d=3$ polymer using OLM. However, we caution the reader that one must be careful before interpreting the linear behaviour. In this regard, we believe that a proper FSS analysis as done for the $d=3$ case is required to confirm it, for which one needs data from different system sizes. This analysis is in progress and will be presented elsewhere.

\subsection{Aging in $d=2$}

We now move on to present some preliminary results on the aging dynamics during polymer collapse in $d=2$ using the OLM. Like in the $d=3$ case here also, we probe aging via calculation of the autocorrelation function described in (4) by using the same criterion for $O_{i}$ as used in $d=3$ for the OLM. To check the presence of aging we first confirm the absence of time-translation invariance. This is demonstrated in Figure 13 for the same system as presented for the cluster growth in Figure 12. The plot shows the autocorrelation function $C\left(t, t_{w}\right)$ as a function of the translated time $t-t_{w}$ for four different values of $t_{w}$ as mentioned in the figure. The absence of time-translation invariance is evident from the non-collapsing behaviour of the data.

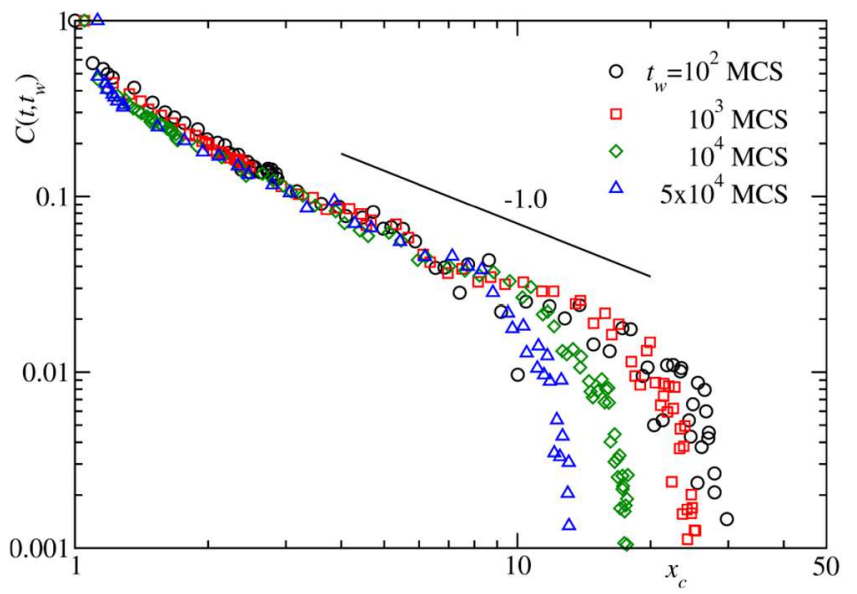

Fig. 14. Illustration of the presence of dynamical scaling of the autocorrelation function shown in Figure 13, plotted here on a double-log scale as a function of the scaling variable $x_{c}=$ $C_{s}(t) / C_{s}\left(t_{w}\right)$. The solid line shows the consistency of the data with a power-law decay having an exponent $\lambda_{c}=1.0$.

Along with that one can also notice that the larger $t_{w}$ the slower the autocorrelation decays which confirms the second criterion of aging, i.e., slow dynamics. The last criterion for aging is the presence of dynamical scaling. In the present case of polymer collapse in $d=2$, unlike in the $d=3$ case with OLM, we do not observe any data collapse with respect to the scaling variable $x_{c}=t / t_{w}$. This, on the other hand, is similar to the results obtained for the LM in $d=3$. However, to limit ourselves here rather than going for an analysis based on subaging scaling we immediately look for the scaling with respect to $x_{c}=C_{s}(t) / C_{s}\left(t_{w}\right)$ and indeed find a reasonable collapse of data implying the presence of simple aging behaviour. This is demonstrated in Figure 14 where we plot $C\left(t, t_{w}\right)$ as a function of $x_{c}=C_{s}(t) / C_{s}\left(t_{w}\right)$ for four different choices of $t_{w}$.

The other important aspect of aging is to quantify the autocorrelation exponent $\lambda_{c}$ for which an idea can be obtained from the double-log plot in Figure 14. There for intermediate values of $x_{c}$, the collapsed data show almost a linear behaviour implying a power-law scaling. The solid line corresponds to the power-law decay in equation (7) with an exponent $\lambda_{c}=1$ that is consistent with the data. For a better quantification of $\lambda_{c}$ one would need to do a FSS analysis by using data from a few larger chain lengths. From the general bound given in equation (30), one can read off the corresponding bound in $d=2$,

$$
0.5 \leq \lambda_{c} \leq 1.0
$$

where we have used the fact that in $d=2$, the Flory exponent is exactly $\nu_{F}=0.75[78,98]$. The consistency of our data in Figure 14 with the autocorrelation exponent $\lambda_{c}=1$ implies that in $d=2$ the bound is marginally obeyed. However, to have an appropriate verification of the bound one needs to have a more reliable estimate of $\lambda_{c}$ as already mentioned. 


\section{Conclusion and outlook}

We have presented an overview of results existing in the literature regarding the collapse dynamics of a homopolymer in $d=3$ dimensions. Although research in this direction started long back with the proposition of the sausage model of collapse by de Gennes, after a series of works by Dawson and co-workers [21-26] and a few other [27-33], it eventually faded away. Particularly, in experiments it was difficult to monitor a single polymer to verify the phenomenological theories developed around collapse dynamics. Recently, motivated by the successful experimental development for monitoring single polymers and polymers in very dilute solutions, we have provided some new insights in the collapse dynamics of polymers via computer simulations. In this regard, we borrowed tools and understanding from the general nonequilibrium process of coarsening in particle and spin systems. This allowed us to explore different nonequilibrium scaling laws that could be associated with kinetics of the collapse transition of polymers.

When speaking of scaling laws concerning collapse dynamics of a polymer the first thing one looks for is the scaling of the overall collapse time $\tau_{c}$ with the chain length $N$ (which was also the main focus of the studies in the past). From a survey of the available results in this direction it is clear that for power-law scaling of the form $\tau_{c} \sim N^{z}$, the value of the dynamical exponent $z$ obtained depends on the intrinsic dynamics used in the simulations. Especially one has to be careful about presence of hydrodynamics while quoting the value of $z$. However, in our work with an off-lattice model via Monte Carlo dynamics for large $N$, we obtained a value of $z$ that is close to the one obtained from molecular dynamics simulations with preservation of hydrodynamic effects. This raises the question of to what extent hydrodynamic interactions are important during collapse. A proper answer to this could be obtained via systematic studies of polymer models with explicit solvent $[34,103,104]$. For the latter there also exist few studies; however, with no consensus about the value of $z$. In the context of doing simulations with explicit solvent it would also be interesting to see the effect of the viscosity of the solvent particles on the dynamics. Building of such a framework is possible with an approach based on the dissipative particle dynamics [105-108]. Recently, we have taken up this task by using an alternative approach to dissipative particle dynamics [109,110]. In this context, we have successfully constructed the set up and tested that it reproduces the correct dynamics in equilibrium taking consideration of the hydrodynamic interactions appropriately [111]. To add more to this understanding recently we have also considered the task of doing all-atom molecular dynamics simulations with explicit solvent [112]. There the focus is on understanding the collapse of a polypeptide in water with the aim to get new insights to the overall folding process of a protein which contains these polypeptides as backbone.

Coming back to the scaling laws during collapse our approach of understanding the collapse in analogy with usual coarsening phenomena allows us to explore the cluster kinetics appropriately. Our findings from studies using both off-lattice and lattice models show that the average cluster size $C_{s}(t)$ during the collapse grows in a powerlaw fashion as $C_{s}(t) \sim t^{\alpha_{c}}$. However, the growth exponent $\alpha_{c}$ is not universal with $\alpha_{c} \approx 1$ for the off-lattice model and $\alpha_{c} \approx 0.62$ for the lattice model. For quantification of this growth exponent one must be careful about the initial cluster formation stage which sets a high off-set while fitting the data to a simple power law. In this regard, we have introduced a nonequilibrium finite-size scaling analysis which helps to estimate the value of $\alpha_{c}$ unambiguously.

Along with the growth kinetics where one deals with single-time quantities, it is also important to have understanding of the multiple-time quantities which provide information about the aging during such nonequilibrium processes. In analogy with the two-time density or order-parameter autocorrelation function used in usual coarsening of particle or spins systems, we have shown how one can construct autocorrelation functions to study aging during collapse of a polymer. Depending on the nature of the model (whether off-lattice or lattice) the chosen observable to calculate the autocorrelation may vary; however, qualitatively they should give the same information. Our results indeed support our choice of the respective observables and provide evidence of aging and corresponding dynamical scaling of the form $C\left(t, t_{w}\right) \sim$ $\left[C_{s}(t) / C_{s}\left(t_{w}\right)\right]^{-\lambda_{c}}$. Unlike the growth exponent, the autocorrelation exponent was found to be $\lambda_{c}=1.25$ irrespective of the nature of the model, implying that the aging behaviour is rather universal. In this regard, it is worth mentioning that even choosing two different bond criteria for the lattice model (one with the diagonal bonds and the other without it [40]) yielded cluster growth exponents that are different, however, the autocorrelation exponent $\lambda_{c}$ still remains universal with a value of 1.25 . To check the robustness of this universality, a study of other polymer models both off-lattice and lattice, along with different methods of simulations as mentioned previously is required.

In addition to the review of the existing results we have also presented preliminary results in the context of polymer collapse in $d=2$ dimensions. To understand a two-dimensional system is not only of fundamental interest [113], but could be of relevance in the context of polymers confined to an attractive surface. Indeed there are experiments of synthetic polymers on two-dimensional gold or silver surfaces $[114,115]$. Our results on the kinetics of polymer collapse in $d=2$ show that the phenomenology associated with this process can still be described by the "pearl-necklace" picture of Halperin and Goldbart, albeit the identification of the small pearl-like clusters which coarsen to form the final globule is not as distinct as in the $d=3$ case. Via an extension of the $d=3$ methodologies to $d=2$, we observe that the cluster formation stage in $d=2$ is rather slow. However, the late-time coarsening of the clusters follows the same power-law scaling $C_{s}(t) \sim t^{\alpha_{c}}$ with $\alpha_{c} \approx 1$. We also have presented results for the aging dynamics in this regard as well. There the autocorrelation function shows the same kind of power-law scaling as in $d=3$ with a corresponding exponent $\lambda_{c} \approx 1$. A more detailed study not only with the off-lattice model but also with the lattice model is in progress. 
Finally, we feel that this novel approach of understanding the collapse dynamics of polymers from the perspective of usual coarsening studies of particle and spin systems shall serve as a general platform which could be used to analyze the nonequilibrium evolution of macromolecules in general across any conformational transition. Of course, due to their distinct features, for each class of this transition the associated techniques shall be modified accordingly. One has to choose the appropriate properties of the system and find out the best quantities that describe the corresponding transition appropriately in nonequilibrium. For example, one can look at the helixcoil transition of macromolecules as well $[116,117]$. There certainly the average cluster size would not work as a suitable quantity to monitor the kinetics. Rather one may define some local helical order parameter and look at the corresponding time dependence.

Open access funding provided by Projekt DEAL. This project was funded by the Deutsche Forschungsgemeinschaft (DFG, German Research Foundation) under project Nos. JA 483/331 and 189853844 - SFB/TRR 102 (project B04), and the Deutsch-Französische Hochschule (DFH-UFA) through the Doctoral College "L $\mathbb{L}^{4}$ " under Grant No. CDFA-02-07. We further acknowledge support by the Leipzig Graduate School of Natural Sciences "BuildMoNa".

\section{Author contribution statement}

S.M. planned the structure of the manuscript with inputs from the co-authors. All the authors contributed equally in writing and developing the text.

Open Access This is an open access article distributed under the terms of the Creative Commons Attribution License (http://creativecommons.org/licenses/by/4.0), which permits unrestricted use, distribution, and reproduction in any medium, provided the original work is properly cited.

Publisher's Note The EPJ Publishers remain neutral with regard to jurisdictional claims in published maps and institutional affiliations.

\section{References}

1. L.D. Landau, E.M. Lifshitz, Statistical Physics (Pergamon Press, London, 1958)

2. H.E. Stanley, Introduction to Phase Transitions and Critical Phenomena (Clarendon Press, Oxford, 1971)

3. A. Onuki, Phase Transition Dynamics (Cambridge University Press, Cambridge, 2002)

4. S. Puri, V. Wadhawan, eds., Kinetics of Phase Transitions (CRC Press, Boca Raton, 2009)

5. P.-G. de Gennes, Scaling Concepts in Polymer Physics (AIP, Melville, New York, 1980)

6. M. Doi, S.F. Edwards, The Theory of Polymer Dynamics (Clarendon Press, Oxford, 1986)

7. J. des Cloizeaux, G. Jannink, Polymers in Solution (Clarendon Press, Oxford, 1990)

8. M. Rubinstein, R.H. Colby, Polymer Physics (Oxford University Press, New York, 2003)
9. W.H. Stockmayer, Macromol. Chem. Phys. 35, 54 (1960)

10. I. Nishio, S.-T. Sun, G. Swislow, T. Tanaka, Nature 281, $208(1979)$

11. C.J. Camacho, D. Thirumalai, Proc. Natl. Acad. Sci. USA 90, 6369 (1993)

12. L. Pollack, M.W. Tate, A.C. Finnefrock, C. Kalidas, S. Trotter, N.C. Darnton, L. Lurio, R.H. Austin, C.A. Batt, S.M. Gruner et al., Phys. Rev. Lett. 86, 4962 (2001)

13. M. Sadqi, L.J. Lapidus, V. Muñoz, Proc. Natl. Acad. Sci. USA 100, 12117 (2003)

14. G. Haran, Curr. Opin. Struct. Biol. 22, 14 (2012)

15. G. Reddy, D. Thirumalai, J. Phys. Chem. B 121, 995 (2017)

16. B. Chu, Q. Ying, A.Y. Grosberg, Macromolecules 28, 180 (1995)

17. B. Schuler, E.A. Lipman, W.A. Eaton, Nature 419, 743 (2002)

18. J. Xu, Z. Zhu, S. Luo, C. Wu, S. Liu, Phys. Rev. Lett. 96, $027802(2006)$

19. M. Tress, E.U. Mapesa, W. Kossack, W.K. Kipnusu, M. Reiche, F. Kremer, Science 341, 1371 (2013)

20. P.-G. de Gennes, J. Phys. Lett. 46, 639 (1985)

21. A. Byrne, P. Kiernan, D. Green, K.A. Dawson, J. Chem. Phys. 102, 573 (1995)

22. E.G. Timoshenko, Yu.A. Kuznetsov, K.A. Dawson, J. Chem. Phys. 102, 1816 (1995)

23. Yu.A. Kuznetsov, E.G. Timoshenko, K.A. Dawson, J. Chem. Phys. 103, 4807 (1995)

24. Yu.A. Kuznetsov, E.G. Timoshenko, K.A. Dawson, J. Chem. Phys. 104, 3338 (1996)

25. Yu.A. Kuznetsov, E.G. Timoshenko, K.A. Dawson, J. Chem. Phys. 105, 7116 (1996)

26. K.A. Dawson, E.G. Timoshenko, Yu.A. Kuznetsov, Physica A 236, 58 (1997)

27. E. Pitard, H. Orland, Europhys. Lett. 41, 467 (1998)

28. L.I. Klushin, J. Chem. Phys. 108, 7917 (1998)

29. A. Halperin, P.M. Goldbart, Phys. Rev. E 61, 565 (2000)

30. N. Kikuchi, A. Gent, J.M. Yeomans, Eur. Phys. J. E 9, $63(2002)$

31. C.F. Abrams, N.K. Lee, S.P. Obukhov, Europhys. Lett. 59, 391 (2002)

32. A. Montesi, M. Pasquali, F.C. MacKintosh, Phys. Rev. E 69, 021916 (2004)

33. N. Kikuchi, J.F. Ryder, C.M. Pooley, J.M. Yeomans, Phys. Rev. E 71, 061804 (2005)

34. T.T. Pham, M. Bajaj, J.R. Prakash, Soft Matter 4, 1196 (2008)

35. J. Guo, H. Liang, Z.G. Wang, J. Chem. Phys. 134, 244904 (2011)

36. S. Majumder, W. Janke, Europhys. Lett. 110, 58001 (2015)

37. S. Majumder, W. Janke, Phys. Rev. E 93, 032506 (2016)

38. S. Majumder, W. Janke, J. Phys.: Conf. Ser. 750, 012020 (2016)

39. S. Majumder, J. Zierenberg, W. Janke, Soft Matter 13, 1276 (2017)

40. H. Christiansen, S. Majumder, W. Janke, J. Chem. Phys. 147, 094902 (2017)

41. S. Majumder, H. Christiansen, W. Janke, J. Phys.: Conf. Ser. 955, 012008 (2018)

42. P.C. Hohenberg, B.I. Halperin, Rev. Mod. Phys. 49, 435 (1977) 
43. D. Frenkel, B. Smit, Understanding Molecular Simulation: From Algorithms to Applications (Academic Press, San Diego, 2001)

44. P.E. Rouse, J. Chem. Phys. 21, 1272 (1953)

45. B.H. Zimm, J. Chem. Phys. 24, 269 (1956)

46. W. Janke, in Order, Disorder and Criticality: Advanced Problems of Phase Transition Theory, edited by $\mathrm{Y}$. Holovatch (World Scientific, Singapore, 2012), Vol. 3, p. 93

47. W. Janke, in Order, Disorder and Criticality: Advanced Problems of Phase Transition Theory, edited by Y. Holovatch (World Scientific, Singapore, 2018), Vol. 5, p. 173

48. A.J. Bray, Adv. Phys. 51, 481 (2002)

49. R. Shimizu, H. Tanaka, Nat. Commun. 6, 7407 (2015)

50. S. Basu, S. Majumder, S. Sutradhar, S.K. Das, R. Paul, Europhys. Lett. 116, 56003 (2017)

51. H. Christiansen, S. Majumder, W. Janke, Phys. Rev. E 99, 011301(R) (2019)

52. W. Janke, H. Christiansen, S. Majumder, J. Phys.: Conf. Ser. 1163, 012002 (2019)

53. F. Corberi, E. Lippiello, P. Politi, J. Stat. Phys. 176, 510 (2019)

54. I.M. Lifshitz, V.V. Slyozov, J. Phys. Chem. Solids 19, 35 (1961)

55. S.M. Allen, J.W. Cahn, Acta Metall. 27, 1085 (1979)

56. E.D. Siggia, Phys. Rev. A 20, 595 (1979)

57. H. Furukawa, Phys. Rev. A 31, 1103 (1985)

58. M. Henkel, M. Pleimling, in Non-Equilibrium Phase Transitions, Ageing and Dynamical Scaling far from Equilibrium (Springer, Heidelberg, 2010), Vol. 2

59. M. Zannetti, in Kinetics of Phase Transitions, edited by S. Puri, V. Wadhawan (CRC Press, Boca Raton, 2009), p. 153

60. J.-P. Bouchaud, in Soft and Fragile Matter: Nonequilibrium Dynamics, Metastability and Flow, edited by M. Cates, M.R. Evans (IOP, Bristol, 2000), p. 285

61. H.E. Castillo, C. Chamon, L.F. Cugliandolo, M.P. Kennett, Phys. Rev. Lett. 88, 237201 (2002)

62. D.S. Fisher, D.A. Huse, Phys. Rev. B 38, 373 (1988)

63. F. Liu, G.F. Mazenko, Phys. Rev. B 44, 9185 (1991)

64. E. Lorenz, W. Janke, Europhys. Lett. 77, 10003 (2007)

65. J. Midya, S. Majumder, S.K. Das, J. Phys.: Condens. Matter 26, 452202 (2014)

66. C. Yeung, M. Rao, R.C. Desai, Phys. Rev. E 53, 3073 (1996)

67. E. Pitard, J.-P. Bouchaud, Eur. Phys. J. E 5, 133 (2001)

68. N.V. Dokholyan, E. Pitard, S.V. Buldyrev, H.E. Stanley, Phys. Rev. E 65, 030801(R) (2002)

69. M. Cloitre, R. Borrega, L. Leibler, Phys. Rev. Lett. 85, 4819 (2000)

70. P. Bursac, G. Lenormand, B. Fabry, M. Oliver, D.A. Weitz, V. Viasnoff, J.P. Butler, J.J. Fredberg, Nat. Mater. 4, 557 (2005)

71. P. Wang, C. Song, H.A. Makse, Nat. Phys. 2, 526 (2006)

72. A. Milchev, A. Bhattacharya, K. Binder, Macromolecules 34, 1881 (2001)

73. J.S. Shaffer, J. Chem. Phys. 101, 4205 (1994)

74. T. Dotera, A. Hatano, J. Chem. Phys. 105, 8413 (1996)

75. I. Carmesin, K. Kremer, Macromolecules 21, 2819 (1988)

76. G. Subramanian, S. Shanbhag, J. Chem. Phys. 129, 144904 (2008)
77. D.P. Landau, K. Binder, A Guide to Monte Carlo Simulations in Statistical Physics (Cambridge University Press, Cambridge, 2009)

78. P.J. Flory, Principles of Polymer Chemistry (Cornell University Press, Ithaca, NY, 1953)

79. S. Majumder, S.K. Das, Europhys. Lett. 95, 46002 (2011)

80. S. Majumder, S.K. Das, Phys. Rev. E 84, 021110 (2011)

81. S. Majumder, S.K. Das, W. Janke, Phys. Rev. E 98, 042142 (2018)

82. S. Majumder, S.K. Das, Phys. Rev. E 81, 050102(R) (2010)

83. S.K. Das, S. Roy, S. Majumder, S. Ahmad, Europhys. Lett. 97, 66006 (2012)

84. S. Majumder, S.K. Das, Phys. Chem. Chem. Phys. 15, 13209 (2013)

85. M.E. Fisher, in Critical Phenomena, edited by M.S. Green (Academic Press, London, 1971), p. 1

86. V. Privman, Finite Size Scaling and the Numerical Simulations of Statistical Systems (World Scientific, Singapore, 1990)

87. V. Privman, M.E. Fisher, Phys. Rev. B 30, 322 (1984)

88. C.K. Hu, C.-Y Lin, J.-A. Chen, Phys. Rev. Lett. 75, 193 (1995)

89. H.J. Hilhorst, J.M.J. van Leeuwen, Phys. Rev. Lett. 47, 1188 (1981)

90. D. Hérisson, M. Ocio, Eur. Phys. J. B 40, 283 (2004)

91. D. Parker, F. Ladieu, J. Hammann, E. Vincent, Phys. Rev. B 74, 184432 (2006)

92. H. Christiansen, S. Majumder, M. Henkel, W. Janke, arXiv:1906.11815 (2019)

93. J. Kurchan, Phys. Rev. E 66, 017101 (2002)

94. R. Paul, G. Schehr, H. Rieger, Phys. Rev. E 75, 030104(R) (2007)

95. H. Park, M. Pleimling, Phys. Rev. B 82, 144406 (2010)

96. N. Clisby, Phys. Rev. Lett. 104, 055702 (2010)

97. N. Clisby, B. Dünweg, Phys. Rev. E 94, 052102 (2016)

98. C. Vanderzande, Lattice Models of Polymers (Cambridge University Press, Cambridge, 1998)

99. M. Wittkop, S. Kreitmeier, D. Göritz, J. Chem. Phys. 104, 3373 (1996)

100. J.M. Polson, M.J. Zuckermann, J. Chem. Phys. 113, 1283 (2000)

101. P. Grassberger, H.-P. Hsu, Phys. Rev. E 65, 031807 (2002)

102. H. Zhou, J. Zhou, Z.C. Ou-Yang, S. Kumar, Phys. Rev. Lett. 97, 158302 (2006)

103. R. Chang, A. Yethiraj, J. Chem. Phys. 114, 7688 (2001)

104. J.M. Polson, M.J. Zuckermann, J. Chem. Phys. 116, 7244 (2002)

105. P.J. Hoogerbrugge, J.M.V.A. Koelman, Europhys. Lett. 19, 155 (1992)

106. P. Espanol, P. Warren, Europhys. Lett. 30, 191 (1995)

107. R.D. Groot, P.B. Warren, J. Chem. Phys. 107, 4423 (1997)

108. P. Español, P.B. Warren, J. Chem. Phys. 146, 150901 (2017)

109. C.P. Lowe, Europhys. Lett. 47, (1999) 145

110. E.A. Koopman, C.P. Lowe, J. Chem. Phys. 124, 204103 (2006)

111. S. Majumder, H. Christiansen, W. Janke, J. Phys.: Conf. Ser. 1163, 012072 (2019)

112. S. Majumder, U.H.E. Hansmann, W. Janke, Macromolecules 52, 5491 (2019)

113. J. Midya, S.K. Das, Phys. Rev. Lett. 118, 165701 (2017) 
114. S. Förster, W. Widdra, J. Chem. Phys. 141, 054713 (2014)

115. S. Förster, E. Kohl, M. Ivanov, J. Gross, W. Widdra, W. Janke, J. Chem. Phys. 141, 164701 (2014)
116. E. Arashiro, J.R. Drugowich de Felício, U.H.E. Hansmann, Phys. Rev. E 73, 040902(R) (2006)

117. E. Arashiro, J.R. Drugowich de Felício, U.H.E. Hansmann, J. Chem. Phys. 126, 045107 (2007) 